Article

\title{
PRIMAL: Page Rank-Based Indoor Mapping and Localization Using Gene-Sequenced Unlabeled WLAN Received Signal Strength
}

\author{
Mu Zhou ${ }^{1, *}$, Qiao Zhang ${ }^{1}$, Kunjie Xu ${ }^{2}$, Zengshan Tian ${ }^{1}$, Yanmeng Wang ${ }^{1}$ and Wei He ${ }^{1}$ \\ ${ }^{1}$ Chongqing Key Lab of Mobile Communications Technology, Chongqing University of Posts and \\ Telecommunications, Chongqing 400065, China; E-Mails: zhangqiao6120@gmail.com (Q.Z.); \\ tianzs@cqupt.edu.cn (Z.T.); 2012210036@stu.cqupt.edu.cn (Y.W.); hewei@cqupt.edu.cn (W.H.) \\ ${ }^{2}$ Ericsson, San Jose, CA 95134, USA; E-Mail: xu.kunjie@ gmail.com \\ * Author to whom correspondence should be addressed; E-Mail: zhoumu @ cqupt.edu.cn; \\ Tel.: +86-139-8385-0201; Fax: +86-23-6248-7993.
}

Academic Editors: Kourosh Khoshelham and Sisi Zlatanova

Received: 29 June 2015 / Accepted: 21 September 2015 / Published: 25 September 2015

\begin{abstract}
Due to the wide deployment of wireless local area networks (WLAN), received signal strength (RSS)-based indoor WLAN localization has attracted considerable attention in both academia and industry. In this paper, we propose a novel page rank-based indoor mapping and localization (PRIMAL) by using the gene-sequenced unlabeled WLAN RSS for simultaneous localization and mapping (SLAM). Specifically, first of all, based on the observation of the motion patterns of the people in the target environment, we use the Allen logic to construct the mobility graph to characterize the connectivity among different areas of interest. Second, the concept of gene sequencing is utilized to assemble the sporadically-collected RSS sequences into a signal graph based on the transition relations among different RSS sequences. Third, we apply the graph drawing approach to exhibit both the mobility graph and signal graph in a more readable manner. Finally, the page rank (PR) algorithm is proposed to construct the mapping from the signal graph into the mobility graph. The experimental results show that the proposed approach achieves satisfactory localization accuracy and meanwhile avoids the intensive time and labor cost involved in the conventional location fingerprinting-based indoor WLAN localization.
\end{abstract}


Keywords: indoor mapping and localization; Allen logic; gene sequencing; graph drawing; page rank

\section{Introduction}

Nowadays, people spend more than $80 \%$ of their time in the indoor environment, where the signal from the Global Positioning System (GPS) is generally difficult to receive. In this circumstance, many indoor localization systems are proposed to guarantee the performance of a variety of location-based services (LBSs), like the guidance of shopping routes, security and healthcare for the elderly, and asset management in warehouses and modern buildings. At the same time, various kinds of techniques have been developed for indoor localization in the recent decade, like Bluetooth [1], ultrasonic wave [2], radio-frequency ID (RFID) [3], ultra-wideband (UWB) [4], visible light communications (VLC) [5-7] and wireless local area networks (WLAN) [8-11]. Among them, the received signal strength (RSS)-based WLAN localization technique is preferred due to the rapid development of WLAN infrastructures and mobile devices, as well as the wide deployment of WLAN, which is selected as one of the primary high-speed access networks in the indoor environment. To the best of our knowledge, trilateration and location fingerprinting are recognized as two of the most representative approaches used in RSS-based indoor WLAN localization. The performance of the trilateration approach suffers from the inaccurate estimation of the distance from each access point (AP) to the receiver [12,13]. The first reason is that the propagation models used for distance estimation cannot always be effective due to the irregular variation of RSS caused by the signal reflection, scattering and diffraction. The second reason is that many indoor areas where the APs are actually located are not reachable. In this case, both the AP and target locations should be estimated simultaneously, and thereby, the precision of distance estimation generally drops dramatically. On the contrary, the location fingerprinting approach is preferred [14-17]. This approach consists of two phases. In the offline phase, the RSSs at a batch of pre-calibrated reference points (RPs) with known physical coordinates are collected and then stored as the location fingerprints into the radio map. In the online phase, the newly-collected RSSs are matched against the radio map to realize the location estimation. However, the time and labor cost involved in the radio map construction increases rapidly as the area increases.

To solve the cost problem and to guarantee the accuracy of RSS-based indoor WLAN localization, we propose a novel page rank-based indoor mapping and localization (PRIMAL) by using the gene-sequenced unlabeled WLAN RSS for simultaneous localization and mapping (SLAM). In concrete terms, we first carry out the observation of the motion patterns of the people in the target environment to construct the mobility graph by using the Allen logic. Second, we rely on off-the-shelf smartphones to collect the WLAN RSSs, which are not labeled with physical coordinates. Third, the concept of gene sequencing is adopted to determine the correlation relations among different RSS sequences, so as to assemble the RSS sequences into a signal graph. Fourth, we utilize the graph drawing approach to exhibit the graphs in a more readable manner. Finally, by using the proposed page rank (PR) algorithm, the mapping from the signal graph into the mobility graph is constructed. After the previous steps, 
the receiver can be located in the area mapped from the node that is matched by the newly-collected RSSs in the signal space.

The rest of this paper is organized as follows. In Section 2, we show some related work on the existing SLAM approaches in the indoor WLAN environment. The steps of the proposed PRIMAL are discussed in detail in Section 3. The extensive experimental results are provided in Section 4. Finally, Section 5 concludes the paper and presents some future directions.

\section{Related Work}

In response to the cost problem faced by location fingerprinting in the conventional RSS-based indoor WLAN localization [18-21], many existing works suggested using motion sensing as a candidate to perform the localization. The authors in [22] collected the WLAN RSSs to construct a logic graph, which can be used to characterize the physical layout of the target environment, and meanwhile rely on an accelerator to explore the reachability among different physical areas, as well as to detect the status of RSSs. After that, based on the constructed mapping relationship between the logic graph and ground-truth graph, the target location is estimated in a specific area for each location query. Using a smartphone, the authors in [23] invented a pedestrian tracking system, which can automatically construct both the floor plan of the anonymous target environment and the corresponding radio map. An indoor tracking system based on the labeled topological map constructed by SLAM is addressed in [24]. See [25]: a foot-mounted inertial measurement unit (IMU) is used to perform proprioceptive motion sensing, and meanwhile, an action recognition system is applied to observe the landmarks of location-related actions. The authors in [26] proposed a pedestrian tracking system by integrating the odometry data collected by the foot-mounted IMU and WLAN RSSs. The localization system developed in [27] is based on the fusion of the image data and data from the IMU in a smartphone. The GraphSLAM approach proposed in [28] is appropriate for a large-scale environment, since there is no signature uniqueness assumption in the GraphSLAM. The authors in [29] constructed a multi-modal signal map from the RSSs collected by all of the available sensors. The work in [30] depended on the IMU sensors to label the RSSs as the pedestrian walks in the same direction. A new concept of the Wi-Fi fingerprint (FP), which considers the order relation among the RSS rather than the absolute values of RSSs, is addressed in [31]. The authors in [32] present a new localization approach, in which the training data are obtained by means of finite difference time domain (FDTD) simulations of electromagnetic propagation.

To deal with the computational complexity problem for localization, the authors in [33] use decision trees to minimize the complexity of the localization system. The authors in [34] rely on the joint clustering technique, which performs the clustering of locations to reduce the computational cost. A new low-complexity tracking scheme is proposed in [35], which is based on Fano's sequential decoding algorithm. The authors in [36] propose the multiple filters (MFs)-based implementation approach, which achieves a significant reduction of the computational complexity. The authors in [37] compare the performance of the probabilistic Gaussian kernel fingerprint-based indoor positioning algorithm by using different types of smartphones. An energy-efficient WLAN-based indoor positioning algorithm, which factors out every part of the probabilistic fingerprint formulae, is proposed in [38]. 
Different from the existing work in the literature, we propose a novel indoor mapping and localization approach, namely the PRIMAL, which is independent of location fingerprinting and motion sensing. Furthermore, there is no requirement of extra infrastructure or devices compared to the conventional approaches. The four main contributions of this paper are summarized as follows. First of all, there is no requirement of location fingerprinting and motion sensing, which saves much time and labor cost. Second, based on people's motion pattern observation, the mobility graph, which is constructed by using the Allen logic, can help greatly in investigating the motion behavior of the people in the target environment. Third, we apply the graph drawing approach to exhibit both the mobility and signal graphs in a more readable manner. Finally, by adopting the PAalgorithm, we conduct the indoor mapping and localization simultaneously. Table 1 summarizes the main symbols used in this paper.

Table 1. Symbol notation.

\begin{tabular}{cc}
\hline Symbols & Description \\
\hline$I_{z}$ & Time duration of the event $z$ \\
$a_{i}$ & The $i$-th RSS vector in sequence $a$ \\
$b_{j}$ & The $j$-th RSS vector in sequence $b$ \\
$H(i, j)$ & Matching score between $a_{i}$ and $b_{j}$ \\
$m$ & Length of sequence $a$ \\
$n$ & Length of sequence $b$ \\
$s\left(a_{i}, b_{j}\right)$ & Similarity function of RSS pair $a_{i}$ and $b_{j}$ \\
$W_{k}$ & Gap scoring function with depth $k$ \\
$\mathbf{R S S}_{l}$ & The $l$-th RSS sequence \\
$\operatorname{rss}_{l i}$ & The $i$-th RSS vector in $\mathbf{R S S}_{l}$ \\
\hline
\end{tabular}

\section{System Description}

\subsection{Construction of the Mobility Graph}

To obtain the connectivity among different areas of interest, we conduct the people's motion pattern observation in the target environment. Figure 1 shows the layout of the six areas of interest, and Figure 2 illustrates the people's 17 motion patterns during a working day. Each motion pattern consists of different events, which are separated by the break points (BPs).

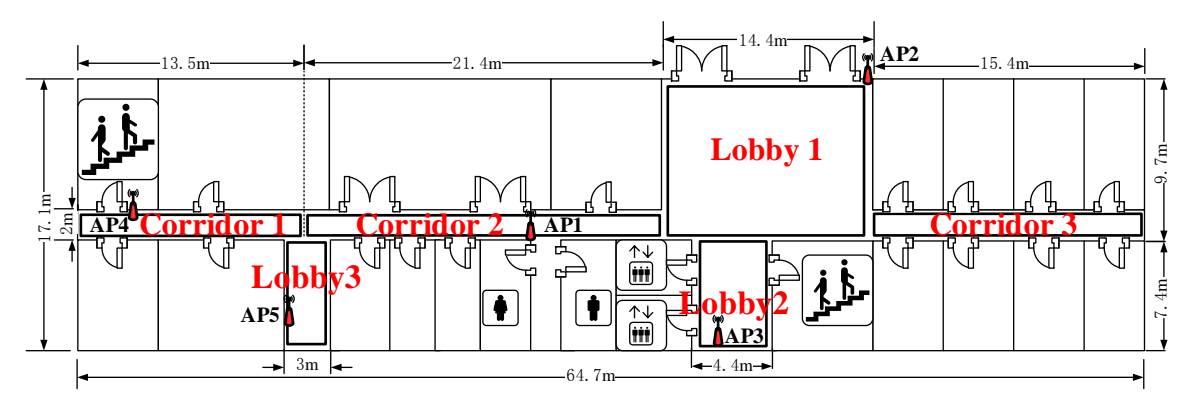

Figure 1. Layout of the target area. 


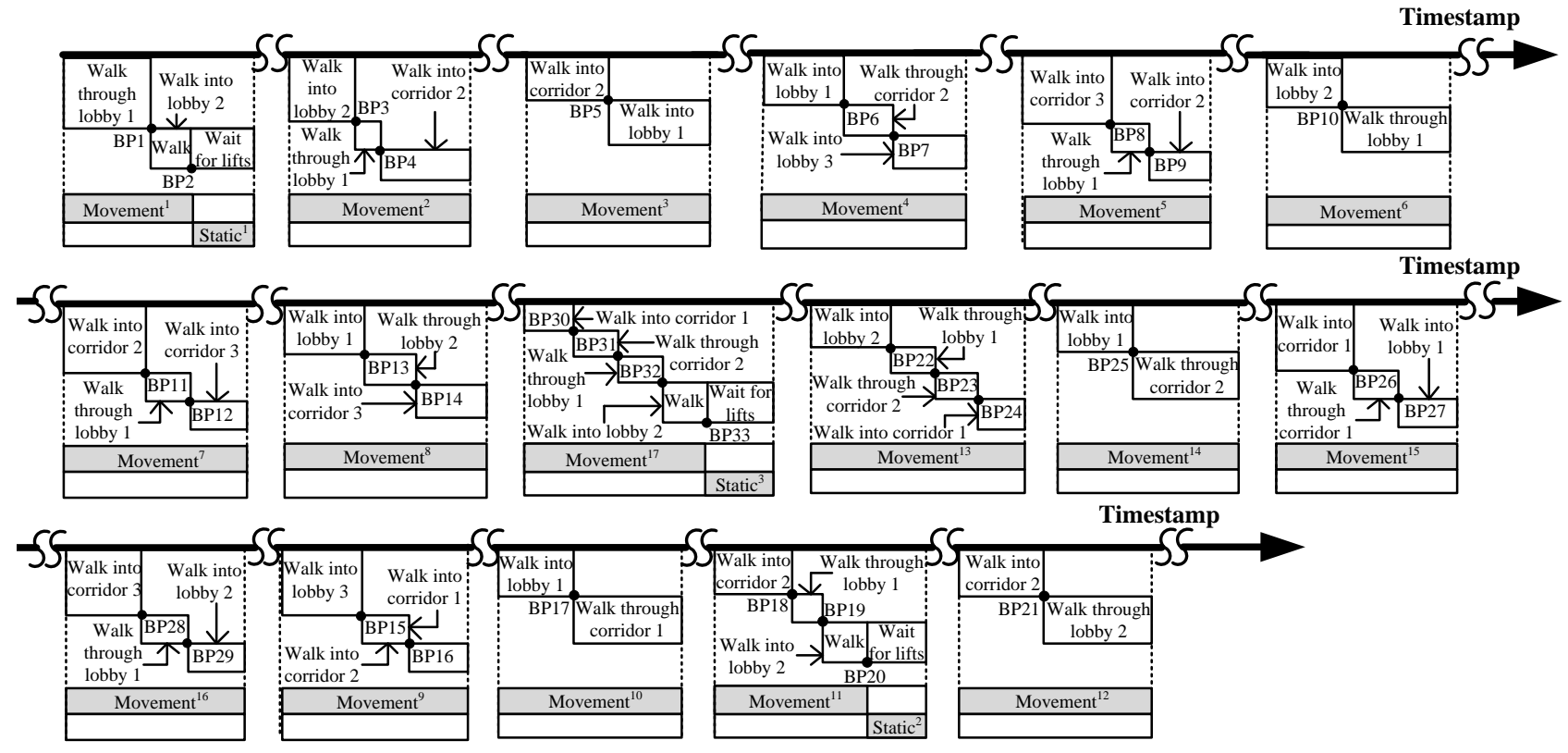

Figure 2. Motion patterns.

In the Allen logic, the relations of events can be represented by 13 different logic operations, as described in Figure 3. On this basis, by using the Allen logic, we draw the event graphs corresponding to the people's 17 different motion patterns in Figure 4. In each graph, we mark the longest path (or the path involving the largest number of hops) with a dashed line. On each longest path, the event nodes are connected by the edges with the "m" operation, while the last even node and movement node are connected by an edge with the " $\mathrm{f}$ " operation. The event nodes that are connected by the edges with the " $m$ " operation indicate the adjacent events happening in chronological order, while the edge with the " $\mathrm{f}$ " operation connecting the final event node and movement node indicates that the last event corresponds to the movement state. Based on this, the event nodes on the longest path can be used to describe the connectivity among different areas of interest. For instance, in the first graph, based on the longest path that is associated with the movement ${ }^{1}$, we obtain a motion behavior between Lobby 1 and Lobby 2 and thereby construct a connection between these two lobbies. Hence, the mobility graph can be constructed by considering all of the motion behaviors of the people in the target environment, as shown in Figure 5 .

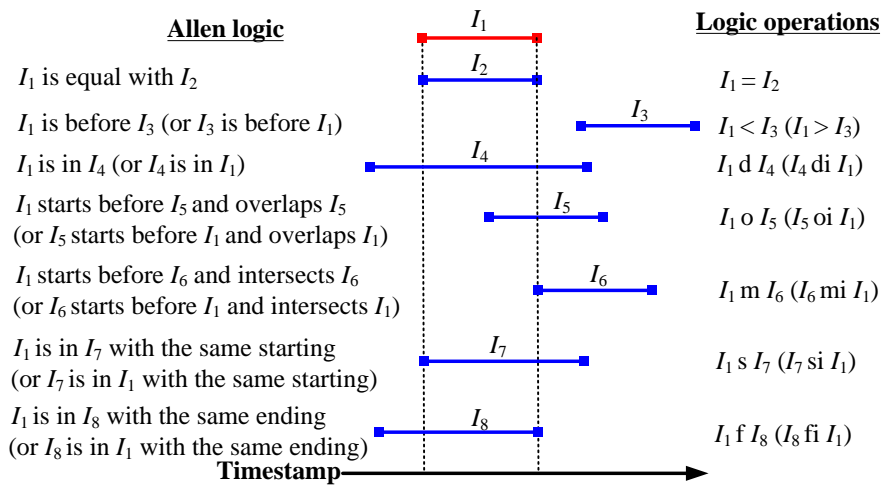

Figure 3. The Allen logic for event expression. 

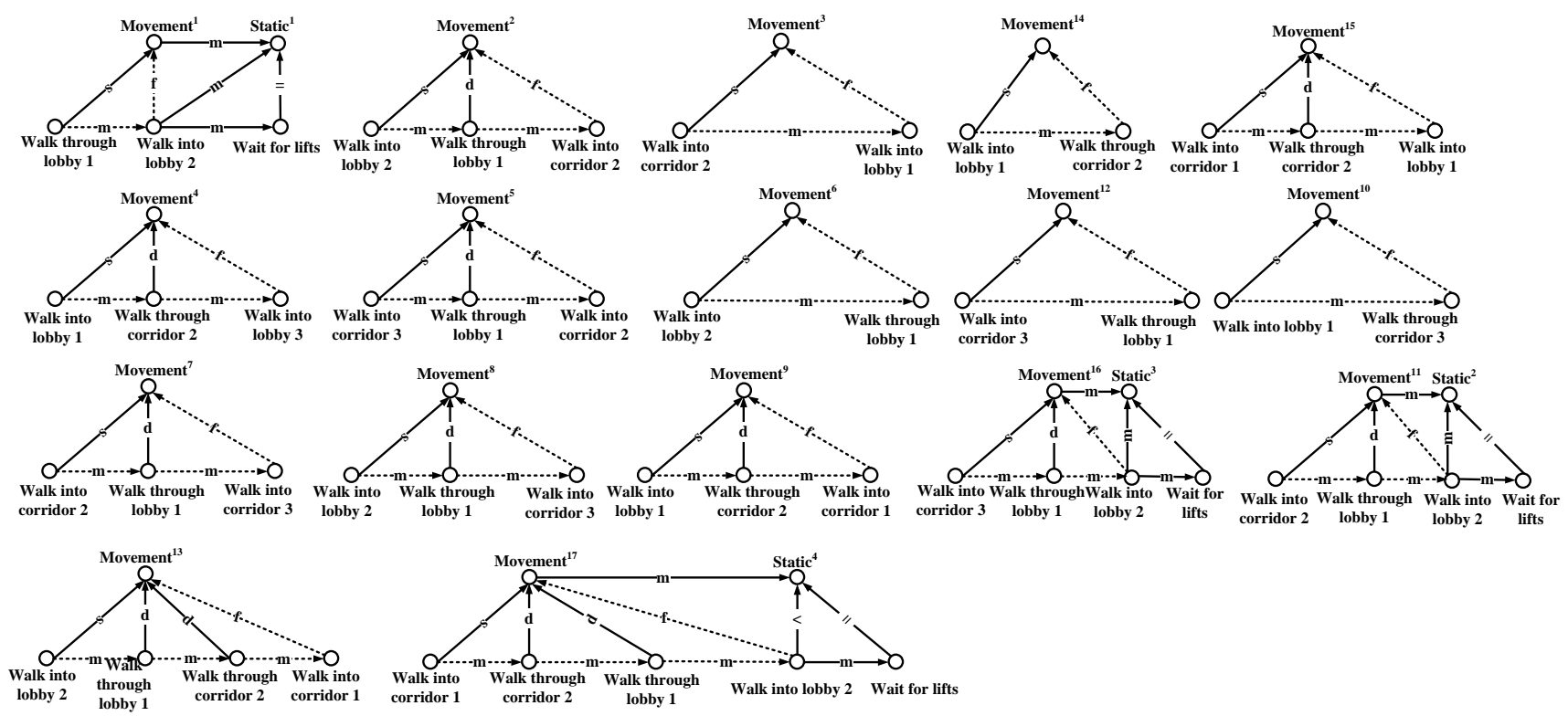

Figure 4. Event graphs.

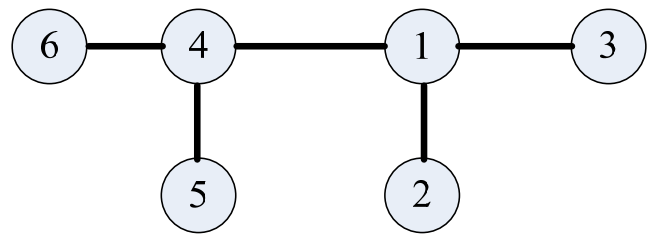
(1) Lobby 1
(2) Lobby 2
(3) Corridor 3
(4) Corridor 2
(5) Lobby 3
(6) Corridor 1
- Connection between the neighboring areas

Figure 5. Mobility graph.

\subsection{Construction of the Signal Graph}

\subsubsection{RSS Characteristics}

A significant reason for using the WLAN RSSs to conduct the SLAM is due to the property that the WLAN RSSs collected in two different areas that are separated by a wall could vary greatly. Figure 6 shows an example of the variations of two WLAN RSS sequences as the signal goes through a wall. We take Sequence 1 (with *'s) as an example. The mean of the RSS before crossing the wall is about $-72 \mathrm{dBm}$, whereas after crossing the wall, it decreases to $-82 \mathrm{dBm}$. Hence, the variation of WLAN RSSs can help with characterizing the layout of the target environment. 


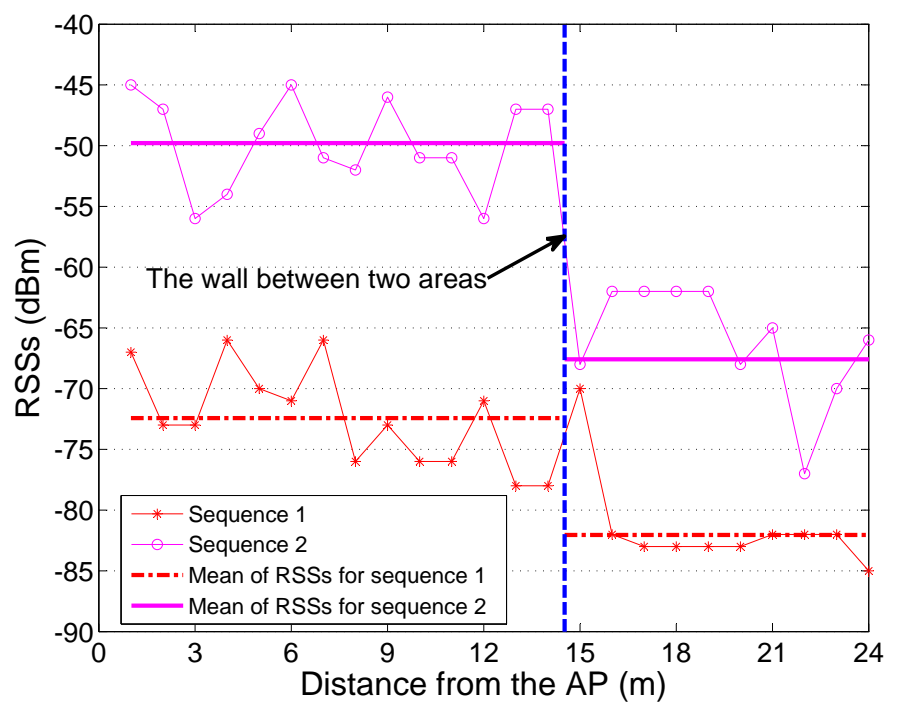

Figure 6. Variation of WLAN RSSs.

\subsubsection{Gene Sequencing}

Based on the people's motion pattern observation during a working day, we obtain the frequencies of the area transitions between every two adjacent areas of interest in Figure 7. There are in total nine patterns of area transitions counted by the path separation, as shown in Figure 8.

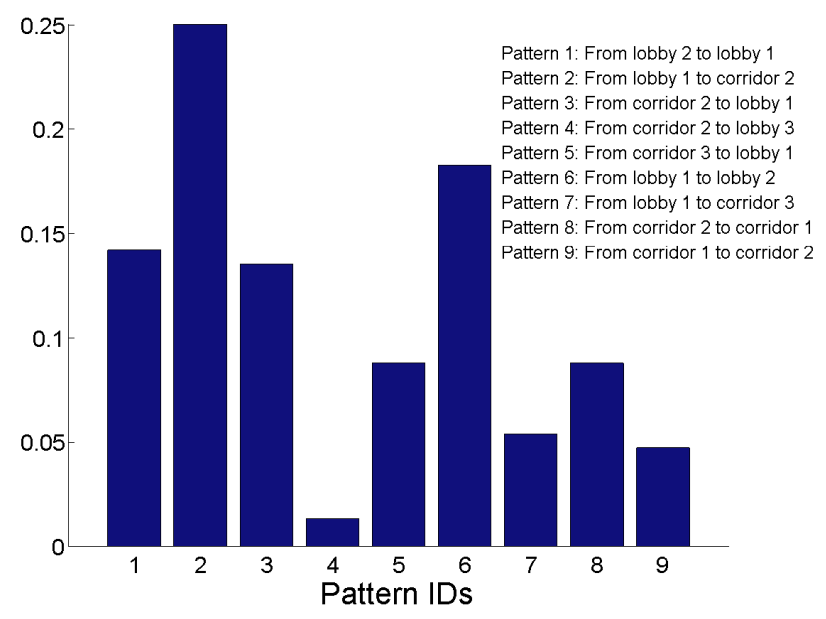

Figure 7. Frequencies of area transitions.

In the target environment, we collected 94 WLAN RSS sequences that obey the frequencies of area transitions shown in Figure 7. Figure 9 illustrates the format of RSS sequences where $k$ is the number of APs, $\operatorname{RSS}_{i j l}$ is the $j$-th RSS vector in the $i$-th RSS sequence from the $l$-th AP and $m$ is the number of RSS vectors in each sequence. Table 2 shows the number of RSS sequences collected on each trace. Hence, the collected RSS sequences can not only reflect the connectivity among different areas of interests, but also depict the motion patterns of the people in the signal space. Therefore, by separating each RSS sequence into different segments of RSSs with high correlation, we can obtain the transitions of segments and consequently assemble the RSS sequences into a signal graph. 


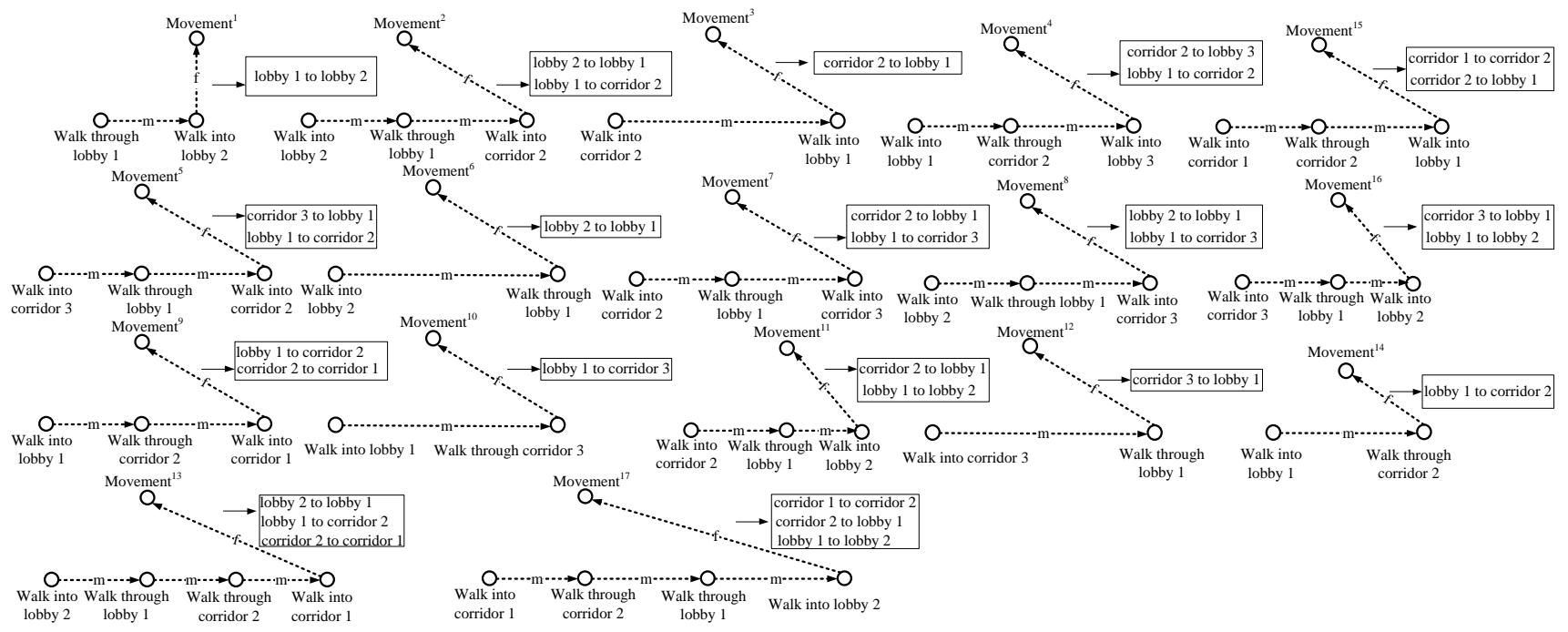

Figure 8. Path separation.

\begin{tabular}{|c|}
\hline$\left(\operatorname{RSS}_{i 11}, \mathbf{R S S}_{i 12}, \cdots, \mathbf{R S S}_{i 1 k}\right)$ \\
\hline$\left(\operatorname{RSS}_{i 21}, \mathbf{R S S}_{i 22}, \cdots, \mathbf{R S S}_{i 2 k}\right)$ \\
\hline$\left(\mathbf{R S S}_{i 31}, \mathbf{R S S}_{i 32}, \cdots, \mathbf{R S S}_{i 3 k}\right)$ \\
\hline$\ldots$ \\
\hline$\left(\right.$ RSS $_{i m 1}$, RSS $_{i m 2}, \cdots$, RSS $\left._{i m k}\right)$ \\
\hline
\end{tabular}

Figure 9. Format of RSS sequences.

Table 2. Number of RSS sequences on each trace.

\begin{tabular}{cc}
\hline Traces & Number of Collected RSS Sequences \\
\hline Lobby $2 \rightarrow$ Lobby 1 & 10 \\
Lobby $2 \rightarrow$ Corridor 2 & 5 \\
Corridor $2 \rightarrow$ Lobby 1 & 7 \\
Lobby $1 \rightarrow$ Corridor $2 \rightarrow$ Lobby 3 & 2 \\
Corridor $3 \rightarrow$ Corridor 2 & 9 \\
Lobby $1 \rightarrow$ Lobby 2 & 19 \\
Corridor $2 \rightarrow$ Corridor 3 & 4 \\
Lobby $2 \rightarrow$ Corridor 3 & 1 \\
Lobby $1 \rightarrow$ Corridor $2 \rightarrow$ Corridor 1 & 8 \\
Lobby $1 \rightarrow$ Corridor 3 & 3 \\
Corridor $2 \rightarrow$ Lobby 2 & 2 \\
Corridor $3 \rightarrow$ Lobby 1 & 2 \\
Lobby $2 \rightarrow$ Corridor $2 \rightarrow$ Corridor 1 & 5 \\
Lobby $1 \rightarrow$ Corridor 2 & 8 \\
Corridor $1 \rightarrow$ Corridor $2 \rightarrow$ Lobby 1 & 3 \\
Corridor $3 \rightarrow$ Lobby 2 & 2 \\
Corridor $1 \rightarrow$ Corridor $2 \rightarrow$ Lobby 2 & 4 \\
\hline
\end{tabular}


In gene sequencing, given two sequences, $a$ and $b$, a scoring matrix, $H$, is constructed to detect the segments of RSSs with high correlation, namely the correlation segments, between these two sequences. The elements in $H$ are calculated by:

$$
\begin{aligned}
& H(i, 0)=0,0 \leq i \leq m, H(0, j)=0,0 \leq j \leq n \\
& 0 \\
& H(i, j)=\max \left\{\begin{array}{c}
H(i-1, j-1)+s\left(a_{i}, b_{j}\right) \\
\max _{k \geq 1}\left\{H(i-k, j)+W_{k}\right\} \\
\max _{l \geq 1}\left\{H(i, j-l)+W_{l}\right\}
\end{array}\right\} \\
& 1 \leq i \leq m, 1 \leq j \leq n
\end{aligned}
$$

where $H(i, j)$ is the matching score between the $i$-th nucleotide in $a, a_{i}$ and the $j$-th nucleotide in $b$, $b_{j}$. Using this concept, in our system, we view the WLAN RSS sequences as the gene sequences, i.e., $a \sim \mathrm{RSS}_{l}=\left\{\operatorname{rss}_{l 1}, \operatorname{rss}_{l 2}, \ldots \operatorname{rss}_{l m}\right\}$ and $b \sim \mathrm{RSS}_{i}=\left\{\operatorname{rss}_{i 1}, \mathrm{rss}_{i 2}, \ldots \mathrm{rss}_{i n}\right\}$, and the RSS vectors as the nucleotides, i.e., $a_{i} \sim \operatorname{rss}_{l i}$ and $b_{j} \sim \operatorname{rss}_{i j}$. Thus, the calculation of the matching score between the RSS vectors equals the one between the corresponding nucleotides. To detect the correlation segments, we require that the matching scores between the RSS vectors satisfy:

- $H(i, j) \geq H(i, j+1)$ as $a_{i}=b_{j}$ and $a_{i} \neq b_{j+1}$

- $H(i, j) \geq H(i+1, j+1)$ as $a_{i}=b_{j}$ and $a_{i+1} \neq b_{j+1}$

- $H(i, j) \leq H(i+1, j+1)$ as $a_{i}=b_{j}$ and $a_{i+1}=b_{j+1}$

- $H(i, j) \geq H(i+1, j+1)$ as $a_{i} \neq b_{j}$ and $a_{i} \neq b_{j+1}$

- $H(i, j) \leq H(i+1, j+1)$ as $a_{i} \neq b_{j}$ and $a_{i+1}=b_{j+1}$

These requirements indicate the characteristics as follows.

(i) If the current RSS vector is matched with an RSS vector, but mismatched with the next one, the matching score of the current RSS pair is not lower than the one of the next pair;

(ii) if the current RSS pair is matched, whereas the next RSS pair is mismatched, the matching score of the current RSS pair is not lower than the one of the next pair;

(iii) if the current RSS pair is matched, while the next RSS pair is also matched, the matching score of the current RSS pair is not higher than the one of the next pair;

(iv) if the current RSS vector is mismatched and still mismatched with the next one, the matching score of the current RSS pair is not lower than the one of the next pair;

(v) if the current RSS pair is mismatched, whereas the next RSS pair is matched, the matching score of the current RSS pair is not higher than the one of the next pair.

To satisfy these requirements, we define the similarity function, $s\left(a_{i}, b_{j}\right)$, and gap scoring function, $W_{k}$, in Equation (2). Based on this, the matching scores satisfy the previous requirements, as proven in the Appendix Section. For the collected 94 RSS sequences, we construct in total 4371 scoring matrices (see Supplementary Material). Figure 10 shows nine of them in which each pixel represents the matching score of an RSS pair, while the larger pixel values indicate the higher similarities between the RSS vectors.

$$
\left\{\begin{array}{c}
s\left(a_{i}, b_{j}\right)=\left\{\begin{array}{c}
\alpha>0, a_{i}=b_{j} \\
\beta<0, a_{i} \neq b_{j}
\end{array}\right. \\
W_{k}=-(\alpha-\beta) k
\end{array}\right.
$$


where $\alpha$ and $\beta$ stand for the reward score and penalty score, respectively.
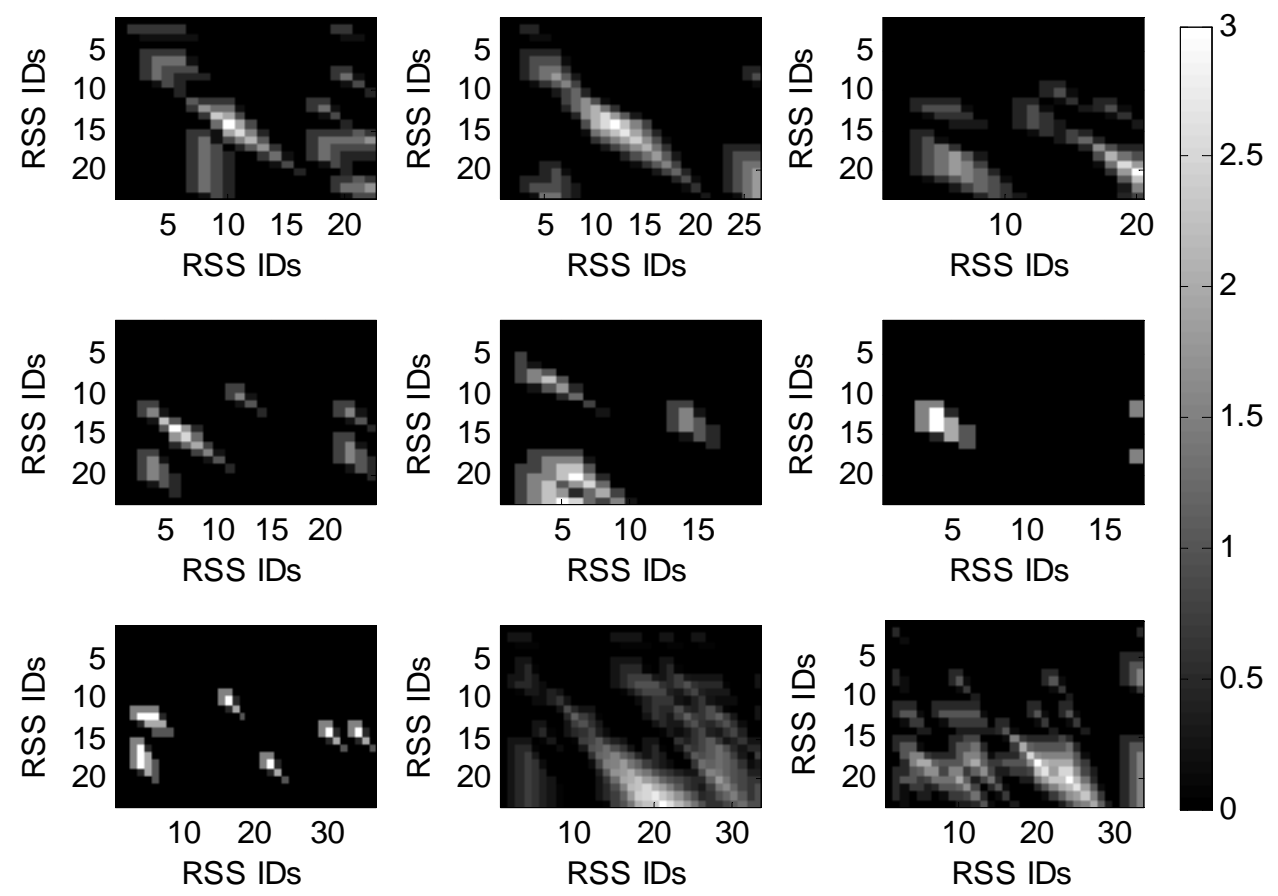

Figure 10. Scoring matrices.

To detect the correlation segments between two RSS sequences, we start from the highest matching score in the corresponding scoring matrix. The steps of this process are described as follows.

Step 1: Locate the highest score in $H$, notated as $H(i, j)$, and then store the location $(i, j)$ into the set $L$;

Step 2: Set $H(i, j)=\max \{H(i-1, j), H(i, j-1), H(i-1, j-1)\}$;

Step 3: Repeat Steps 1 and 2 until we obtain $H(i, j)=0$. We notate $L(r)(1 \leq r \leq g b)$ as the $r$-th location in $L$, and $g b$ is the number of locations stored in $L$;

Step 4: Set $r=g b$;

Step 5: Examine the jump relation between the locations $L(r)$ and $L(r-1)$. If there is a diagonal jump [39] from $L(r)$ to $L(r-1)$, the vertical and horizontal coordinates of $L(r-1)$ are selected to indicate the IDs of the RSS pair with high correlation;

Step 6: Set $r=r-1$;

Step 7: Repeat Steps 5 and 6 until $r$ decreases to two.

Figure 11 gives an example of the scoring matrices with respect to a pair of the same RSS sequences and a pair of RSS sequences collected in different areas, respectively. Obviously, based on the result of the diagonal jumps, which are marked with red rectangles in Figure 12, we detect the segments with high correlation. Hence, we can combine the RSS sequences into different clusters, while the RSSs in the same cluster are featured with high correlation. 


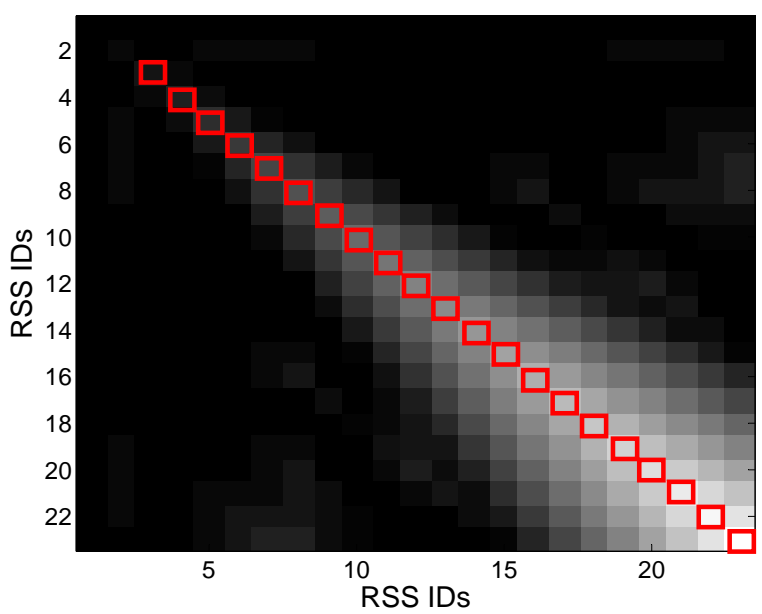

(a)

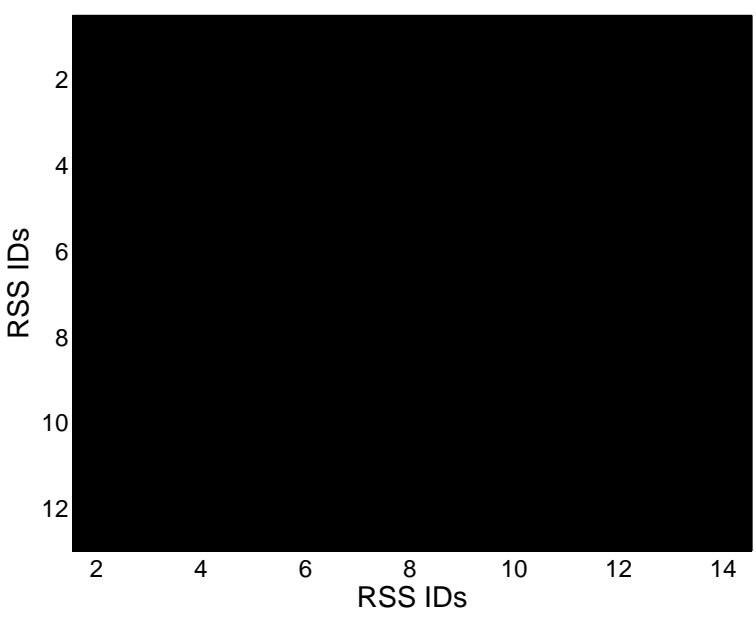

(b)

Figure 11. (a) A pair of the same RSS sequences; (b) a pair of different RSS sequences. Scoring matrices with respect to the same and different RSS sequences.
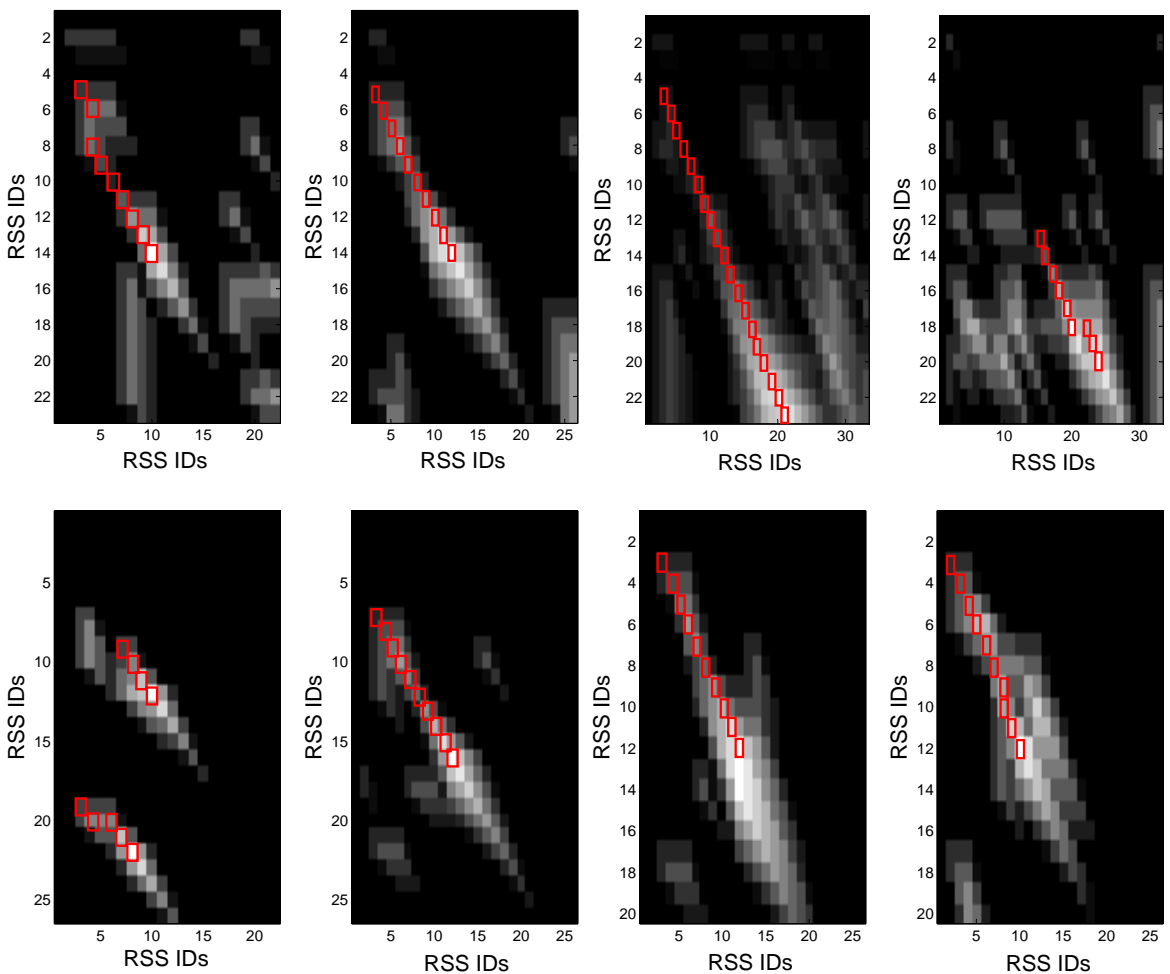

Figure 12. Result of diagonal jumps.

Figure 13 shows the result of RSS sequence combination. There are in total 19 clusters. Based on the transition relations of different clusters, we can assemble the RSS sequences into a signal graph in which each node represents a cluster, while each edge represents the transition relation between two neighboring clusters. The signal graph finally constructed by using the gene sequencing is shown in Figure 14. 


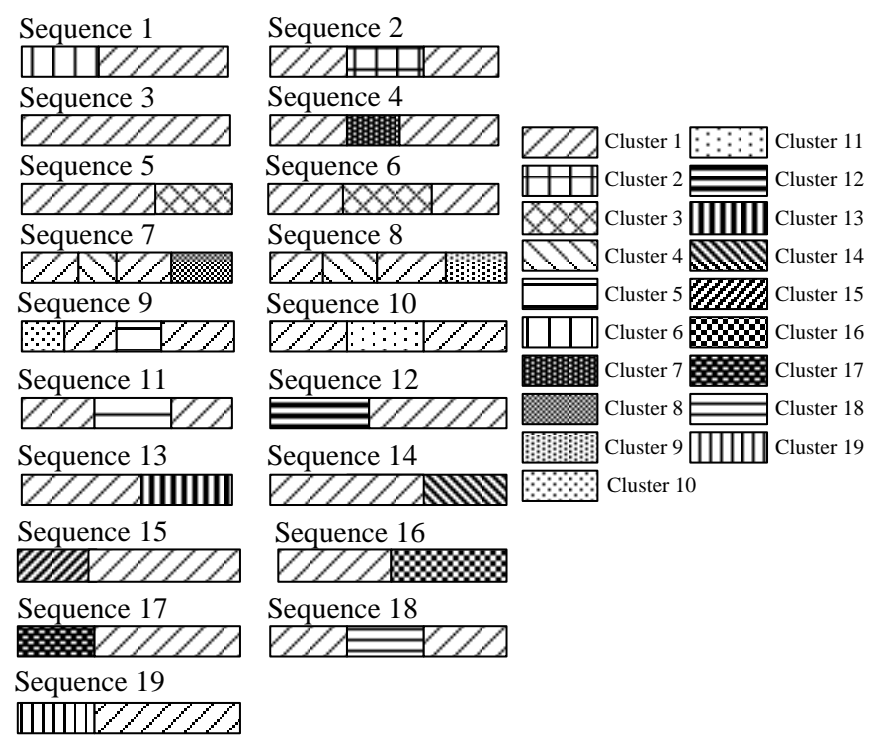

Figure 13. Result of RSS sequence combination.

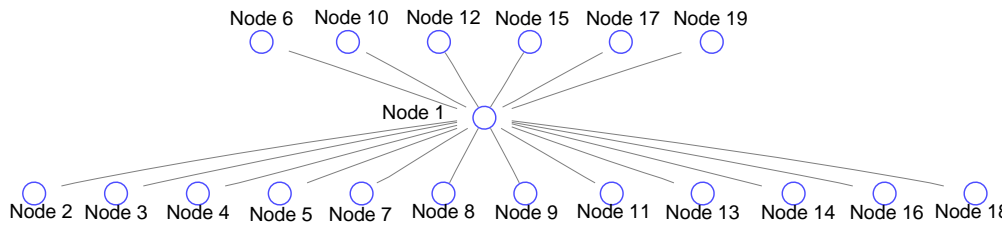

Figure 14. Signal graph.

\subsection{Graph Exhibition by Graph Drawing}

In graph theory, during the visual exhibition of graphs, we can obtain many different layout structures with respect to a given graph, namely the isomorphic graphs. The readable, unique and clear graph exhibition can help greatly in exploring the relations between the graphs in the graph visualization aspect. To achieve this goal, we apply the graph drawing approach to guarantee the uniqueness of the layout structures of the mobility and signal graphs with the purpose of avoiding the confusion of the isomorphic graphs.

Specifically, give a undirected graph $G=(V, E)$, we denote $V=\left\{v_{1}, v_{2}, \ldots, v_{n d}\right\}$ and $E=\left\{\ldots, e_{i j}, \ldots\right\}$ as the sets of vertices and edges. $n d$ is the number of vertices, while $e_{i j}$ indicates that the vertices $v_{i}$ and $v_{j}(i, j \in\{1,2, \ldots, n d\})$ are connected by an edge. The steps of the process of graph drawing are described as follows.

Step 1: Construct the set of vertices, $V_{s}=\left\{\ldots, v_{i}, \ldots\right\}(i \in\{1,2, \ldots, n d\})$, in which the vertices have the same smallest degrees. The degree of vertex $v_{i}, d_{i}$, is defined as the number of vertices connected with $v_{i}$. If there are two vertices $v_{i}$ and $v_{j}$ in $V_{s}$ satisfying the relations that for any vertex $v_{r}(r \in\{1,2, \ldots, n d\})$ in $V-\left\{v_{i}, v_{j}\right\}$, there is at least one path connecting $v_{i}$ and $v_{j}$, i.e., $v_{i} e_{i k} v_{k} \cdots v_{r} e_{r t} v_{t} \cdots v_{j}$, we denote $v_{i}$ and $v_{j}$ as $v_{s}$ and $v_{t}$, respectively, and the set of all the other vertices, $V_{s}-\left\{v_{i}, v_{j}\right\}$, as $v_{s}^{\prime}$. Otherwise, we select another vertex with the smallest degree in $V-V_{s}, v_{t^{\prime}}$. Then, we denote $v_{i}$ and $v_{t^{\prime}}$ as $v_{s}$ and $v_{t}$, respectively, and the set $V_{s}-\left\{v_{i}\right\}$ as $v_{s}^{\prime}$. 
Step 2: After the vertices $v_{s}, v_{s}^{\prime}$ and $v_{t}$ are obtained, we continue to construct a direct graph $G^{\prime}$ from $G$. In concrete terms, for each vertex $v_{i}(i \in\{1,2, \ldots, n d\})$ in $V-\left\{v_{s}, v_{s}^{\prime}, v_{t}\right\}$, we construct the set of all direct paths as $P A_{i}=\left\{\left(v_{s} e_{s y} v_{y} \ldots e_{r i} v_{i} \ldots v_{j} e_{j t} v_{t}\right) \cup\left(v_{s}^{\prime} e_{s y^{\prime}} v_{y^{\prime}} \ldots e_{r^{\prime} i} v_{i} \ldots v_{j^{\prime}} e_{j^{\prime} t} v_{t}\right) \cup \ldots\right\}=$ $\left\{P_{a_{1}} \cup P_{a_{2}} \cup \ldots P_{a_{t}}\right\}\left(y, y^{\prime}, r, r^{\prime}, j, j^{\prime} \in\{1,2, \ldots, n d\}\right)$ starting from $v_{s}$ (or $v_{s}^{\prime}$ ) to $v_{t}$ and containing $v_{i}$, where $t$ is the number of paths in $P A_{i}$ and $P_{a_{j}}(j \in\{1,2, \ldots, t\})$ is the $j$-th path in $P A_{i}$. After that, the set of direct edges, $E_{D}$, can be easily obtained from $P A_{i}$. A direct edge $e_{i j} \in E_{D}$ exists as there is a path $P_{a_{j}} \in P A_{i}$ satisfying $e_{i j} \in P_{a_{j}}$.

Step 3: Label the vertices $v_{i}(i \in\{1,2, \ldots, n d\})$ in $G^{\prime}$, notated as number $\left(v_{i}\right)$, based on the criteria as follows. We set: (i) number $\left(v_{s}\right)=0$, number $\left(v_{s}^{\prime}\right)=0$ and number $\left(v_{j}\right)=\operatorname{number}\left(v_{i}\right)+1$ for the vertices $v_{i}$ and $v_{j}$ on the direct edge $e_{i j}$; and (ii) number $\left(v_{k}\right)=\operatorname{number}\left(v_{i}\right)+1$ and number $\left(v_{j}\right)=\operatorname{number}\left(v_{k}\right)+1$ for $\left\{e_{i j}, e_{i k}, e_{k j}\right\} \subset E_{D}$.

Step 4: Eliminate the edges on $P a_{1}$, which starts from $v_{s}$ (or $v_{s}^{\prime}$ ) to $v_{t}$ and passes by the largest number of edges from $G^{\prime}$. We repeat this process until all of the edges have been eliminated from $G^{\prime}$ or there is no remaining path starting from $v_{s}$ (or $v_{s}^{\prime}$ ) to $v_{t}$. If there are remaining edges after the elimination process, we recognize each remaining edge as a distinct path. After that, we continue to detect all of the internal faces of $G,\left\{F_{h}\right\}(h=1,2, \ldots, \eta)$, where $\eta$ is the number of internal faces. An internal face is defined as a closed region (or the region containing no edge) with the boundary consisting of the edges in the graph. The external face of $G, F$, has the boundary, $C$, containing all of the nodes in $G$, notated as $C=\left(e_{i j} v_{j} e_{j r} \ldots v_{s} e_{s l} \ldots v_{t} e_{t u} \ldots e_{x y} v_{y} e_{y i}\right)(i, j, r, l, u, x, y \in\{1,2, \ldots, n d\})$. We divide $F$ into two sub-regions, $F_{s}$ and $F_{t}$, where $F_{s}$ and $F_{t}$ satisfy the relation $F_{s} \cap F_{t}=\left\{v_{s}, v_{t}\right\}$.

Step 5: Construct a undirected graph $U=\left(V_{U}, E_{U}\right)$, where $V_{U}=\left\{P a_{i}\right\}_{i=1}^{\lambda} \cup\left\{F_{h}\right\}_{h=1}^{\eta} \cup\left\{F_{s}, F_{t}\right\}$ $=\left\{v_{U_{1}}, v_{U_{2}}, \ldots, v_{U_{d}}\right\}, E_{U}=\left\{e_{i j}\right\} \cup\left\{e_{l h}\right\}\left(i, h \in\left\{U_{\lambda+1}, U_{\lambda+2}, \ldots, U_{\lambda+\eta}, U_{\lambda+\eta+1}, U_{\lambda+\eta+2}\right\}, j, l \in\right.$ $\left.\left\{U_{1}, U_{2}, \ldots, U_{\lambda}\right\}\right)$, and $\lambda$ is the number of detected paths in Step 4. We notate the sets of vertices involved in $\left\{P a_{i}\right\}_{i=1}^{\lambda},\left\{F_{h}\right\}_{h=1}^{\eta}$ and $\left\{F_{s}, F_{t}\right\}$ as $\left\{v_{U_{1}}, v_{U_{2}}, \ldots, v_{U_{\lambda}}\right\},\left\{v_{U_{\lambda+1}}, v_{U_{\lambda+2}}, \ldots, v_{U_{\lambda+\eta}}\right\}$ and $\left\{v_{U_{\lambda+\eta+1}}, v_{U_{\lambda+\eta+2}}\right\}$, respectively. We regard $P a_{i}(1 \leq i \leq \lambda)$ as a path vertex, $v_{U_{j}}(1 \leq j \leq \lambda)$. Similarly, $v_{U_{j}}(\lambda+1 \leq j \leq$ $\lambda+\eta+2)$ is regarded as a face vertex. The number of vertices in $U$ equals $d=\lambda+\eta+2$. $e_{i j}$ determines whether there is an edge intersection between the boundaries of the faces of $\left\{F_{h}\right\}_{h=1}^{\eta} \cup\left\{F_{s}, F_{t}\right\}$ and $\left\{P a_{i}\right\}_{i=1}^{\lambda}$. For simplicity, we notate $v_{U_{\lambda+\eta+1}}$ and $v_{U_{\lambda+\eta+2}}$ as $v_{U_{s}}$ and $v_{U_{t}}$.

Step 6: Denote $U, v_{U_{s}}$ and $v_{U_{t}}$ as $G, v_{s}$ and $v_{t}$, respectively, and then, construct the direct graph $U^{\prime}=\left(V_{U^{\prime}}, E_{U^{\prime}}\right)$ from $U=\left(V_{U}, E_{U}\right)$ based on Step 2.

Step 7: Label the vertices in $U^{\prime}$ based on the criteria as follows. We set: (i) number $\left(v_{U_{s}}\right)=-0.5$ and number $\left(v_{j}\right)=\operatorname{number}\left(v_{i}\right)+0.5$ for the vertices $v_{U_{i}}$ and $v_{U_{j}}(i, j \in\{1,2, \ldots, d\})$ on $e_{i j}$; and (ii) $\operatorname{number}\left(v_{k}\right)=\operatorname{number}\left(v_{i}\right)+0.5$ and number $\left(v_{j}\right)=\operatorname{number}\left(v_{k}\right)+0.5$ for $\left\{e_{i j}, e_{i k}, e_{k j}\right\} \subset E_{U^{\prime}}$.

Step 8: Determine the coordinates of the vertices and edges in $G$ based on the criteria as follows. (i) For each vertex, $v_{i}(1 \leq i \leq n d)$, the $Y$ coordinate is the assigned value of $v_{i}$ in $G$, while the range of $X$ coordinates is from the minimum to maximum values assigned to the path associated with $v_{i}$ in $U^{\prime}$; and (ii) for each edge, $e_{i j}(i, j \in\{1,2, \ldots, n d\})$, the $X$ coordinate is the assigned value of $e_{i j}$ in $U^{\prime}$, while the range of $Y$ coordinates is from the minimum to maximum values assigned to the vertices associated with $e_{i j}$ in $G^{\prime}$. 
Figure 15 shows the results of graph drawing for the mobility graph and the signal graph, respectively. From this figure, we observe that by applying the graph drawing approach, the layout structure of graphs becomes unique and more readable in the visualization aspect.

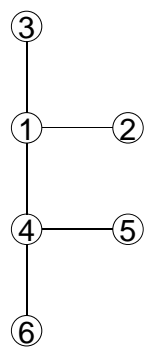

(a)

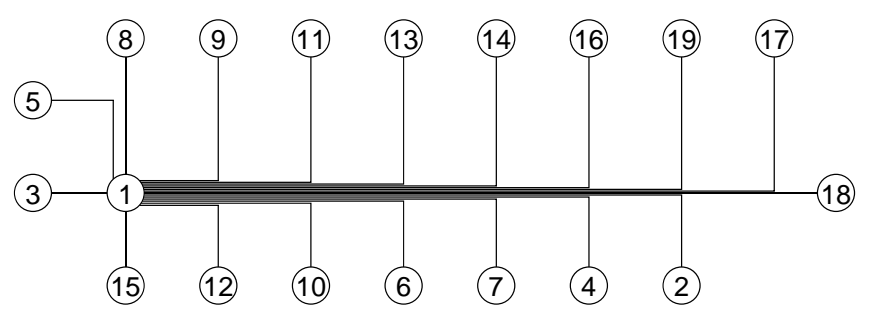

(b)

Figure 15. (a) Mobility graph; (b) signal graph. Results of graph drawing.

\subsection{Page Rank Algorithm}

After the mobility and signal graphs are obtained, we propose to use the PR algorithm to construct the mapping from the signal graph into the mobility graph with the purpose of investigating the relation between the physical layout and signal distribution in the target environment.

First of all, based on the results of path separation in Figure 8, we focus on the detection of the hot areas, which appear frequently in the people's motion patterns. To achieve this goal, we define $P_{z}(i)$ as the probability that the individual has visited the $i$-th area at the timestamp $z$. Thus, we have:

$$
P_{z+1}(i)=\sum_{j} P_{z}(j)\left(P_{j \rightarrow i} / N_{j}\right)
$$

where $N_{j}$ is the number of paths starting from the $j$-th area; and $P_{j \rightarrow i}$ is the area indicator function calculated as follows.

$$
P_{j \rightarrow i}=\left\{\begin{array}{l}
1 \text { when there is a path starting from the } j \text {-th area to } i \text {-th area } \\
0 \text { otherwise }
\end{array}\right.
$$

Second, by assuming that there are $g$ areas in the target environment, we can obtain:

$$
\boldsymbol{P}_{z+1}=\boldsymbol{M} \boldsymbol{P}_{z}
$$

where $\boldsymbol{P}_{z}=\left[P_{Z}(1), P_{Z}(2), \ldots, P_{Z}(g)\right]^{\mathrm{T}}$; and $\boldsymbol{M}$ is a $g \times g$ matrix in which the element on the $i$-th row and $j$-th column is calculated as $m_{i j}=P_{j \rightarrow i} / N_{j}(1 \leq i, j \leq g)$.

In $\boldsymbol{M}, m_{i j}(1 \leq i \leq g)$ becomes zero when $P_{j \rightarrow i}(1 \leq i \leq g)$ is zero, which indicates that there is no path starting from the $j$-th area involved in the people's motion patterns. In this case, we name the current area as the hung area and then distribute the same probability to all of the areas of interest at the next timestamp. On this basis, we modify the matrix $\boldsymbol{M}$ into:

$$
\boldsymbol{S}=\boldsymbol{M}+\boldsymbol{e c}^{\mathrm{T}} / g
$$


where $\boldsymbol{e}=[\underbrace{1,1, \ldots, 1}_{g \text { in total }}]^{\mathrm{T}}$; and $\boldsymbol{c}=\left[c_{1}, c_{2}, \ldots, c_{g}\right]^{\mathrm{T}}$ is an indicator vector in which each element, $c_{i}(1 \leq i \leq g)$, is calculated by:

$$
c_{i}=\left\{\begin{array}{cc}
1 & \text { when the } i \text {-th area is a hung area } \\
0 & \text { otherwise }
\end{array}\right.
$$

After this modification, we can find that the sum of each column in $S$ equals one, which indicates that the individual must appear in one of the areas of interest at the next timestamp. Due to the observation constraint, the area where the individual appears at the next timestamp may not be detected. To solve this problem, we set a probability factor, $\theta$, to describe the probabilities that the individual appears nearby or in other areas at the next timestamp. Hence, we continue to modify the matrix $\boldsymbol{S}$ into:

$$
\boldsymbol{G}=\theta \boldsymbol{S}+(1-\theta) \boldsymbol{e}^{\mathrm{T}} / g
$$

Based on Equation (8), we can find that $\boldsymbol{G}$ is a primitive matrix. Since:

$$
\boldsymbol{P}_{z+1}=\boldsymbol{G} \boldsymbol{P}_{z} \rightarrow \boldsymbol{P}_{z}=\boldsymbol{G}^{z} \boldsymbol{P}_{0}
$$

we calculate the probability distribution of areas as $\boldsymbol{P}=\lim _{Z \rightarrow \infty} P_{Z}$. By setting $\boldsymbol{P}_{0}=[\underbrace{1 / g, 1 / g, \ldots, 1 / g}_{g \text { in total }}]^{\mathrm{T}}$, which indicates that each area has the same probability to be visited at the first timestamp, we can detect the hot areas, which have been visited frequently based on the $\boldsymbol{P}$. Similarly, the probability distribution of clusters in the signal graph can also be calculated based on the results of cluster separation in Figure 16. Table 3 shows the probabilities of the areas and clusters, respectively, in the mobility graph (MG) and the signal graph (SG), respectively.

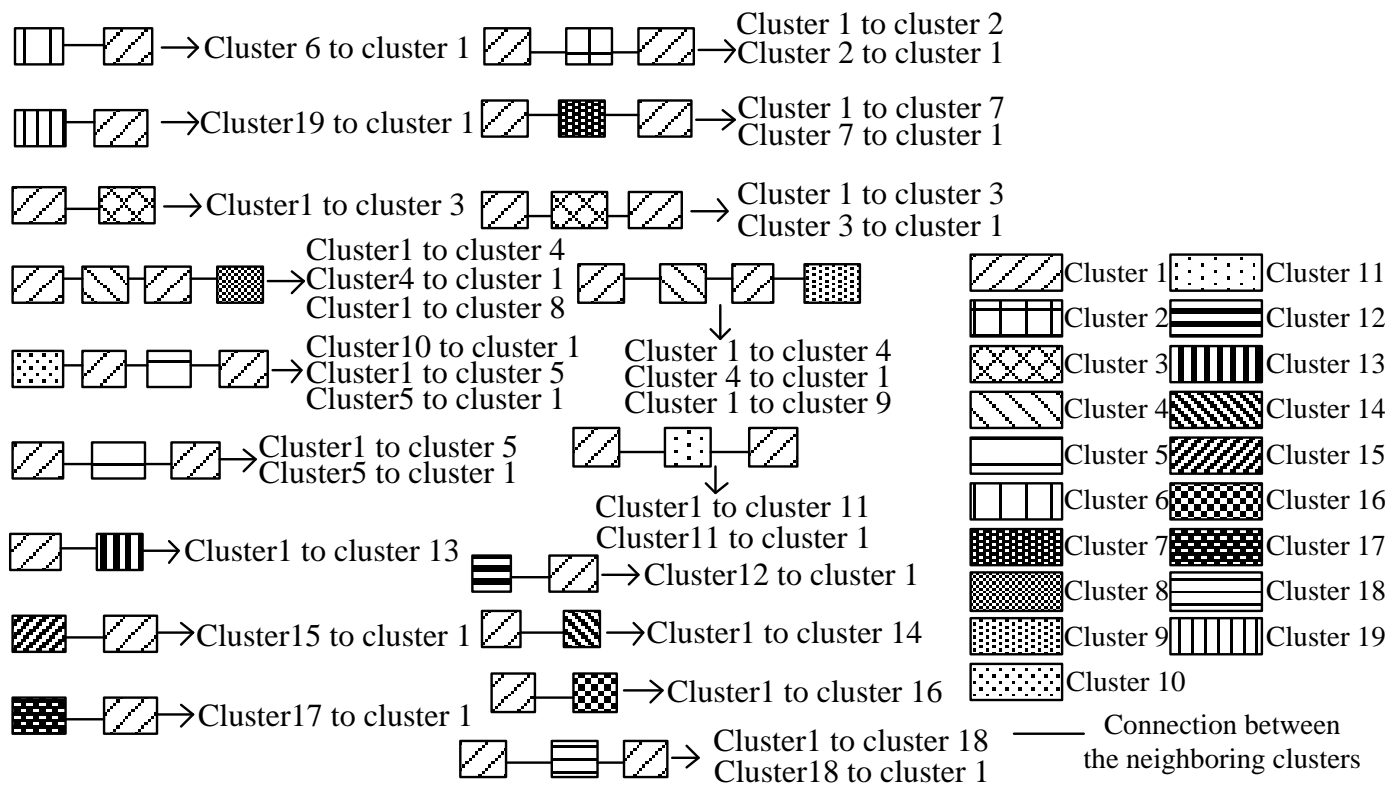

Figure 16. Cluster separation. 
Table 3. Results of probability distributions. MG, mobility graph; SG, signal graph.

\begin{tabular}{ccccccccc}
\hline IDs in MG & $\mathbf{1}$ & $\mathbf{2}$ & $\mathbf{3}$ & $\mathbf{4}$ & $\mathbf{5}$ & $\mathbf{6}$ & \\
\hline Probabilities & 0.27 & 0.11 & 0.05 & 0.22 & 0.03 & 0.09 & \\
IDs in SG & 1 & 2 & 3 & 4 & 5 & 6 & 7 \\
Probabilities & 0.19 & 0.03 & 0.026 & 0.03 & 0.026 & 0.01 & 0.02 \\
IDs in SG & 8 & 9 & 10 & 11 & 12 & 13 & 14 \\
Probabilities & 0.02 & 0.02 & 0.01 & 0.02 & 0.01 & 0.02 & 0.02 \\
IDs in SG & 15 & 16 & 17 & 18 & 19 & & \\
Probabilities & 0.01 & 0.02 & 0.01 & 0.02 & 0.01 & & \\
\hline
\end{tabular}

Finally, we construct the mapping from the signal graph into the mobility graph based on the PR values of the areas and clusters, as shown in Figure 17. In concrete terms, we map the clusters into the areas with the same rank of PR values to preserve the consistency of the hot nodes in the mobility and signal graphs. In our experiments, Area 5 (i.e., Lobby 3) cannot be mapped by any cluster, which means that this area is not a hot area, and meanwhile, there are very few RSSs collected in this area. Therefore, by using the PR algorithm, we not only construct the relation between the signal graph and the mobility graph, but also detect the hot areas that appear frequently in the people's motion patterns.

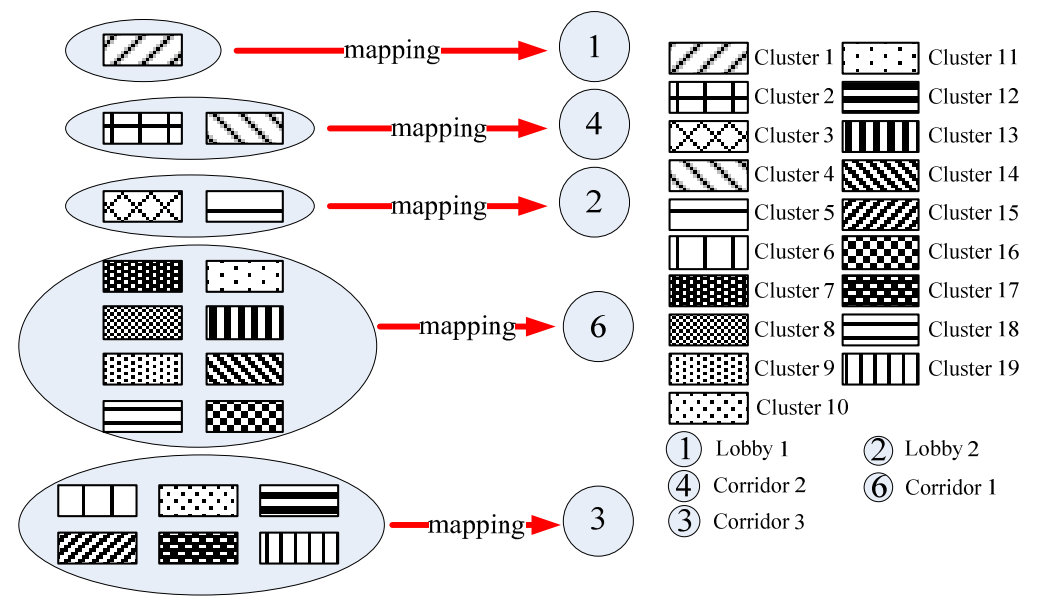

Figure 17. Mapping from the signal graph into the mobility graph.

\subsection{Target Localization}

After the mapping from the signal graph into the mobility graph is constructed, we conduct the localization by using the Kullback-Leibler (KL) divergence. Specifically, first of all, we calculate the distribution of RSS value $i$ in each area, $Q_{j}^{r}(i)$, where $r(1 \leq r \leq g)$ is the area $\operatorname{ID,} j(1 \leq j \leq f)$ is the AP ID and $f$ is the AP number. Second, we calculate the KL divergence between the distribution of the newly-collected RSSs, $T(i)$, and the RSS distribution with respect to each area, $D_{\mathrm{KL}}\left(T \| Q^{r}\right)$, by:

$$
D_{\mathrm{KL}}\left(T \| Q_{j}^{r}\right)=\prod_{j=1}^{f}\left(\sum_{i=1}^{\eta_{r}} T(i) \operatorname{In} \frac{T(i)}{Q_{j}^{r}(i)}\right)
$$

where $\eta_{r}$ is the maximum of RSSs.

Finally, we locate the target in the area that corresponds to the smallest KL divergence. 


\section{Performance Evaluation}

\subsection{Localization Accuracy}

Based on the mapping relation between the signal and mobility graphs, we can obtain the RSS distribution with respect to each area, as shown in Figures 18-22. In our experiments, we find that the number of RSSs mapped into Lobby 1 is much larger than the number of RSSs mapped into the other areas, which means that Lobby 1 is a hot area that appears frequently in the people's motion patterns, as expected. Figure 23 shows the result of localization accuracy. In this figure, the value on the $i$-row and $j$-th column represents the probability of the RSSs in the $i$-th area that have been estimated in the $j$-th area. As can be seen from Figure 23, the probabilities of correct area localization for Areas 1, 2, 3, 4 and 6 approach 100\%, 85\%, 64\%, 64\% and 80\%, respectively. For the RSSs that have been wrongly estimated, the estimated areas are rather adjacent to the correct one, which means that the proposed approach is featured with satisfactory localization accuracy. We take Area 5 as an example. Although all of the RSSs in this area have been wrongly estimated in Area 6, Areas 5 and 6 are rather physically adjacent.

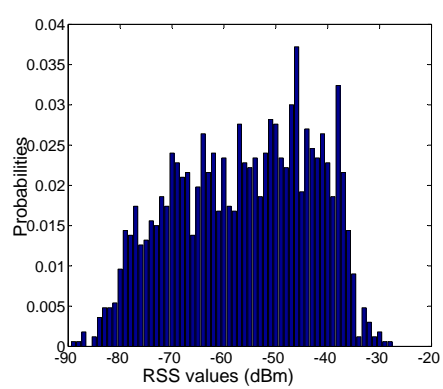

(a)

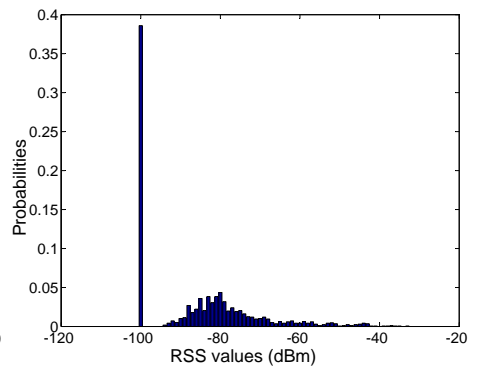

(b)

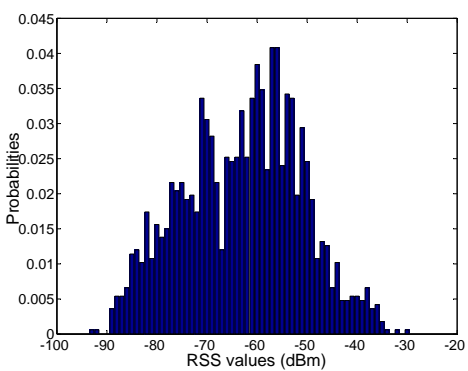

(c)

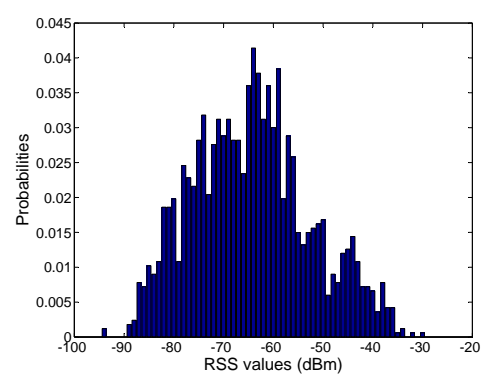

(d)

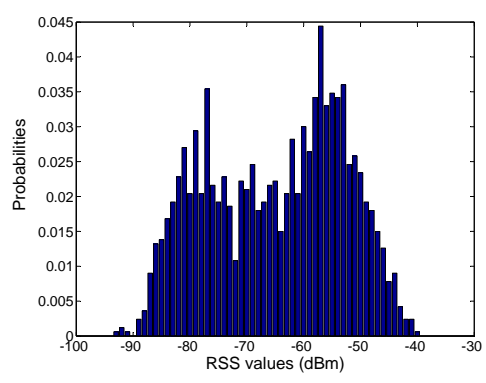

(e)

Figure 18. (a) From Access Point 1 (AP1); (b) from AP2; (c) from AP3; (d) from AP4; (e) from AP5. RSS distributions in Lobby 1. 


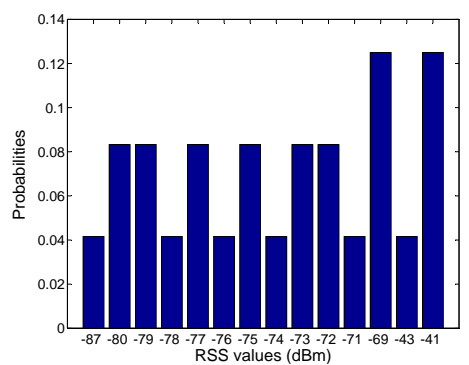

(a)

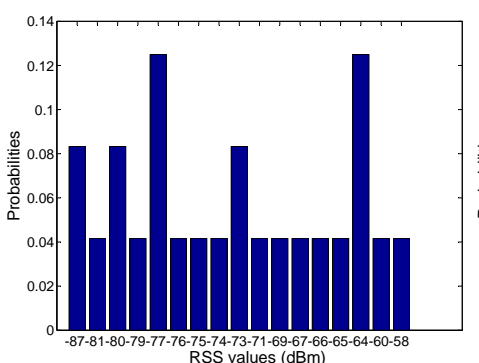

(b)

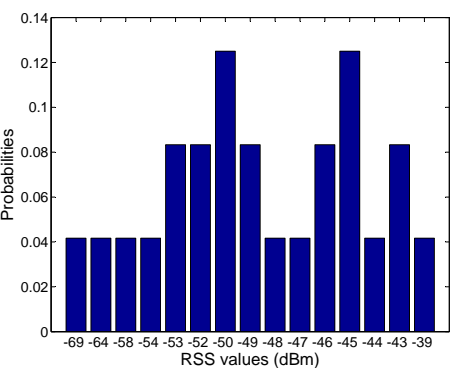

(c)

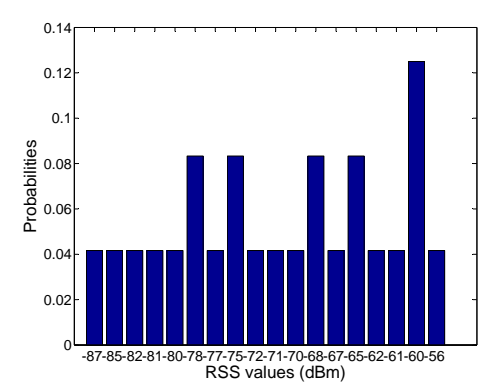

(d)

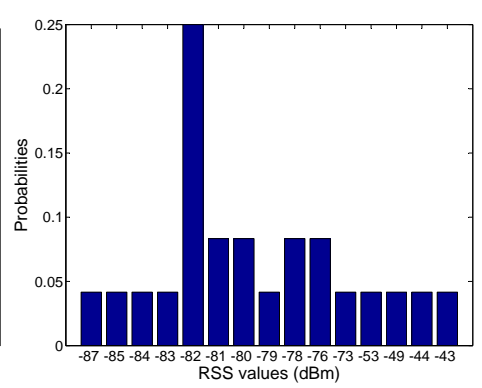

(e)

Figure 19. (a) From AP1; (b) from AP2; (c) from AP3; (d) from AP4; (e) from AP5. RSS distributions in Corridor 2.

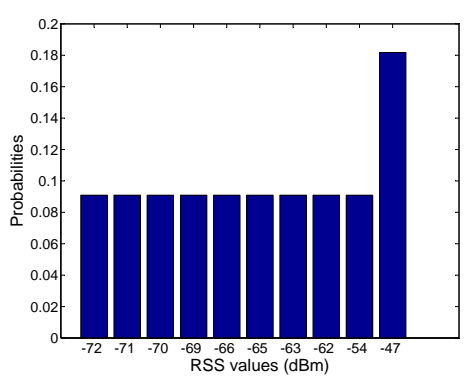

(a)

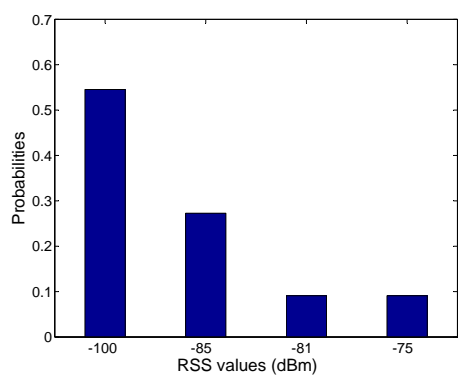

(b)

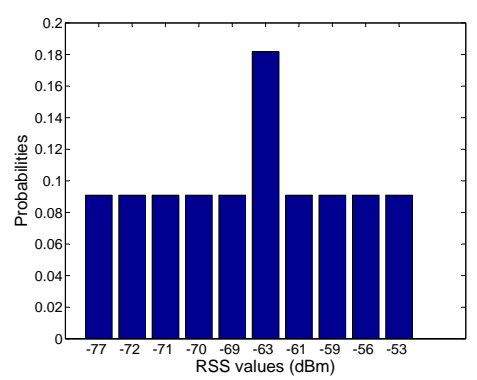

(c)

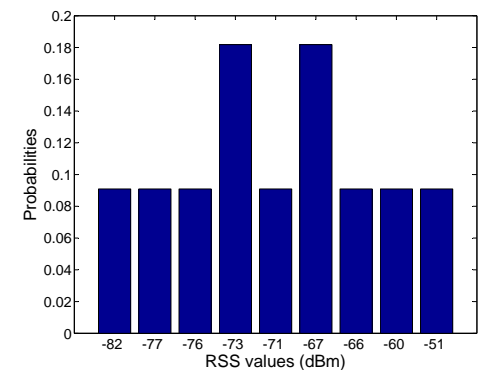

(d)

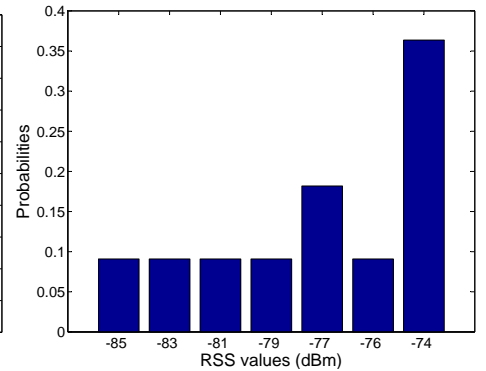

(e)

Figure 20. (a) From AP1; (b) from AP2; (c) from AP3; (d) from AP4; (e) from AP5. RSS distributions in Lobby 2. 


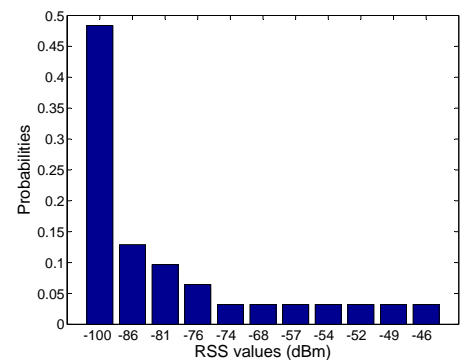

(a)

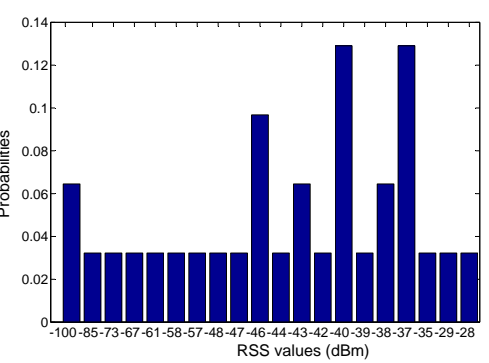

(b)

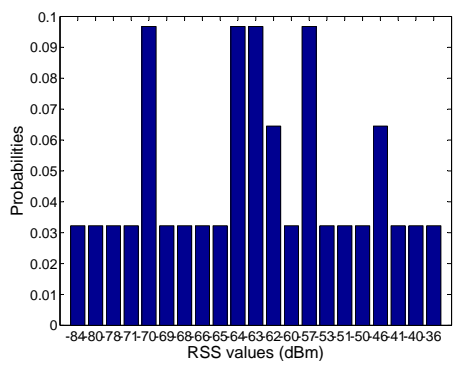

(c)

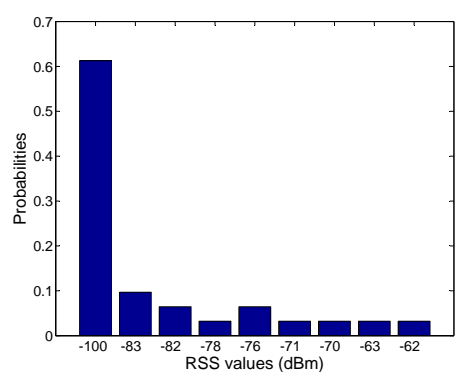

(d)

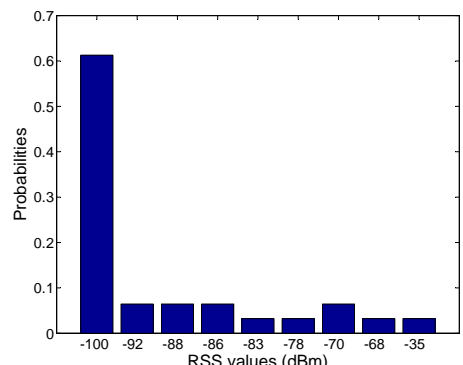

(e)

Figure 21. (a) From AP1; (b) From AP2; (c) From AP3; (d) From AP4; (e) From AP5.RSS distributions in corridor 1 .

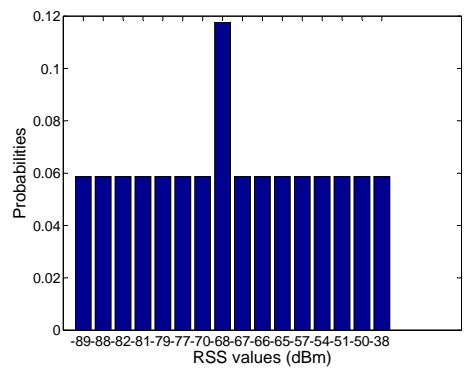

(a)

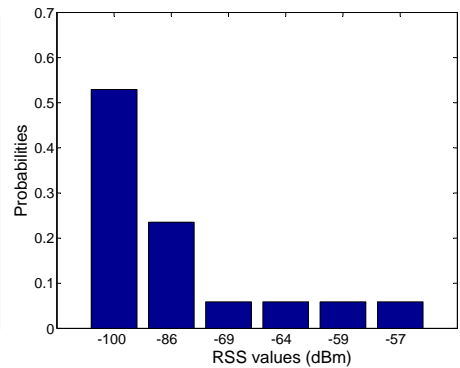

(b)

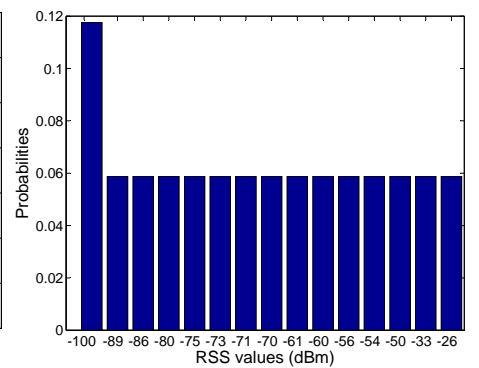

(c)

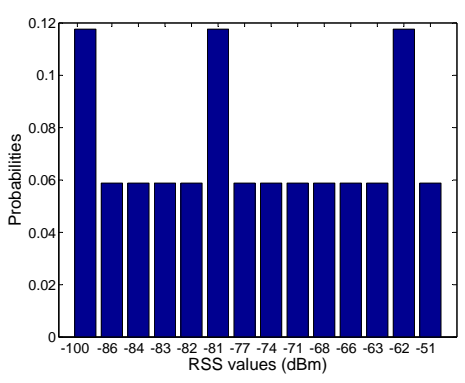

(d)

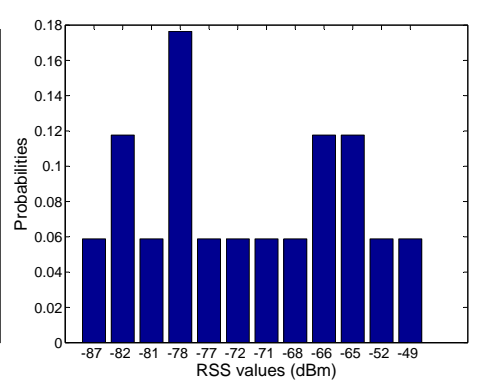

(e)

Figure 22. (a) From AP1; (b) from AP2; (c) from AP3; (d) from AP4; (e) from AP5. RSS distributions in Corridor 3. 


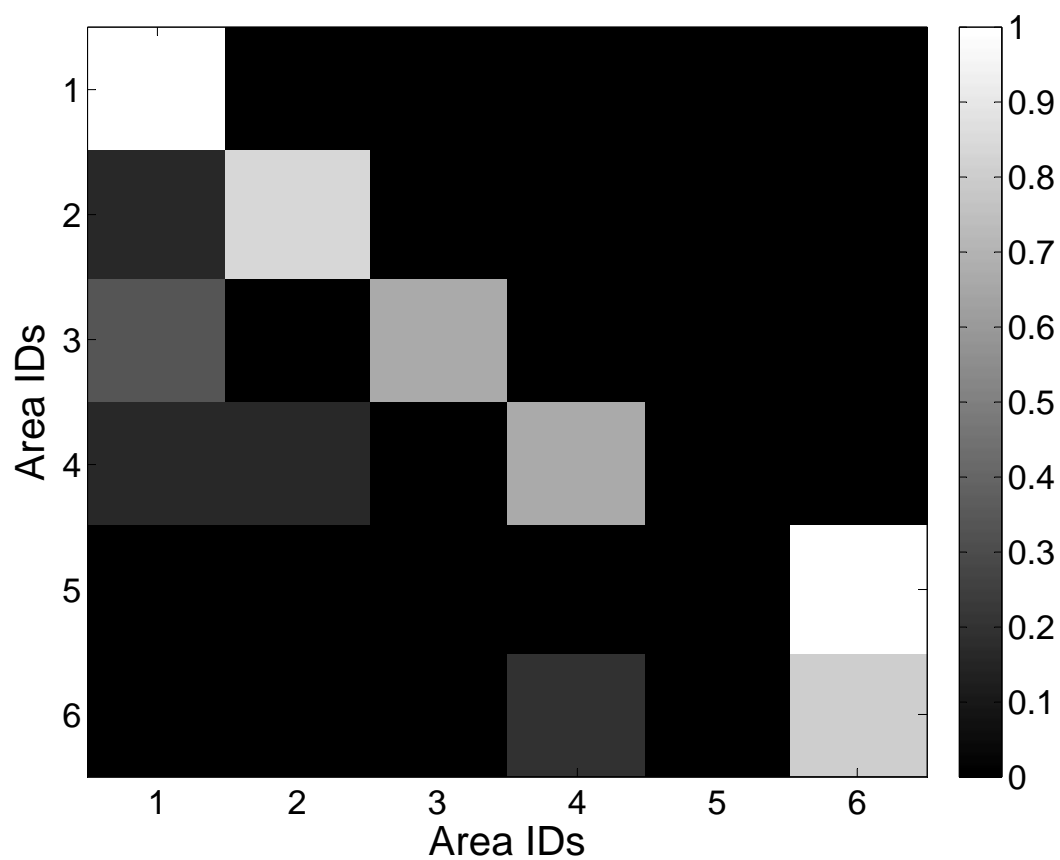

Figure 23. Probabilities of area localization.

\subsection{Parameter Discussion}

Based on Equations (8) and (9), we can find that the value $\boldsymbol{P}$ is determined by the parameter $\theta$. To illustrate this result more clearly, Figures 24 and 25 show the variations of PR values for the mobility graph and signal graph, respectively, under different values of $\theta$. From these figures, we observe that $\theta$ has a slight impact on the rank of PR values. Therefore, we conclude that the value of $\boldsymbol{P}$ seriously relies on the calculation of $S$, which means that the localization and mapping performance is significantly influenced by the observation on the motion patterns of the people in the target environment.

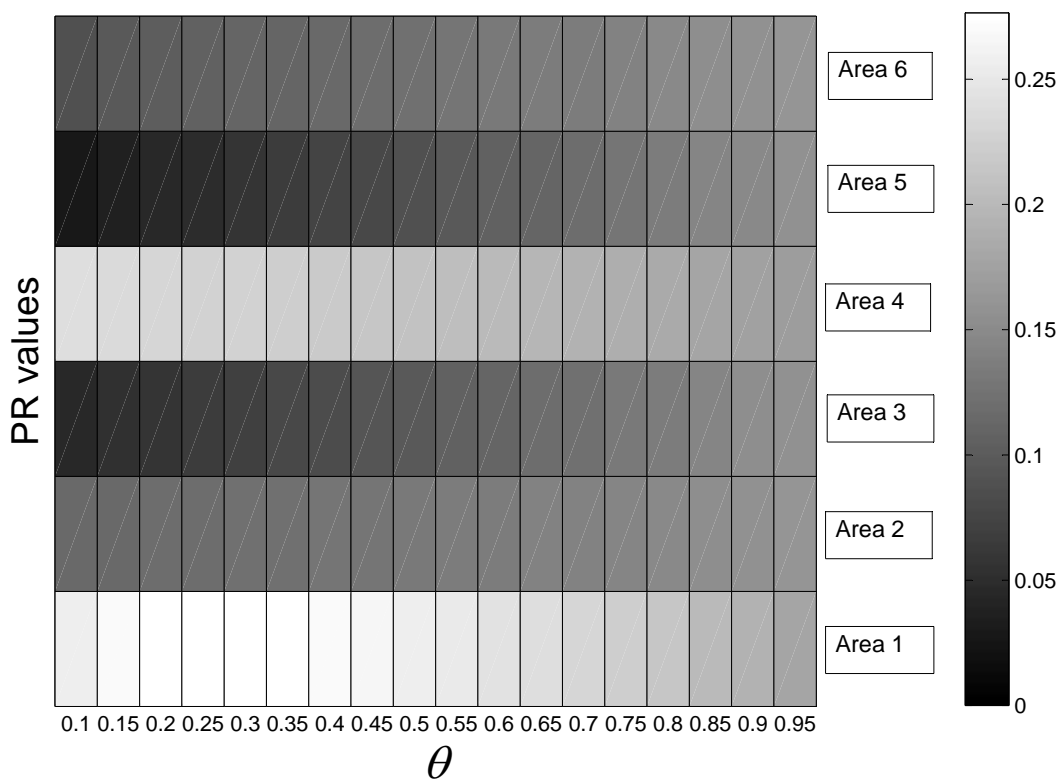

Figure 24. Page rank (PR) values under different values of $\theta$ for the mobility graph. 


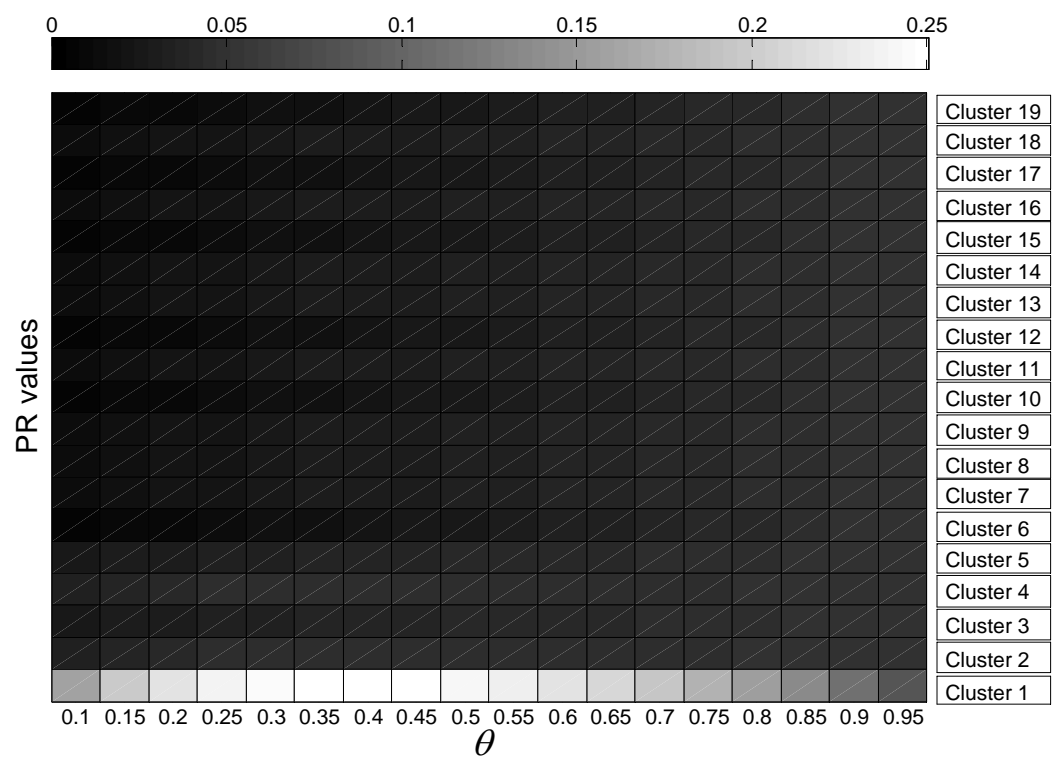

Figure 25. PR values under different values of $\theta$ for the signal graph.

\section{Conclusions and Future Work}

In this paper, we propose to use a novel indoor SLAM approach, namely the PRIMAL, to characterize the layout of the target environment and consequently achieve area-level localization accuracy. Compared to the existing SLAM approaches, the PRIMAL is independent of location fingerprinting and motion sensing. In addition, the PRIMAL can not only detect the hot areas that have been visited frequently by people, but also conduct effective mapping from the signal graph into the mobility graph. Furthermore, with the help of the Allen logic, we rely on the concept of gene sequencing to investigate the correlation between different RSS sequences and meanwhile use the PR algorithm to rank the nodes in the mobility and signal graphs for better understanding of the people's motion patterns in both the physical and signal space. For future work, how to explore the people's motion patterns in a large-scale environment, as well as how to locate the target at the coordinate level form two interesting topics.

\section{Supplementary Materials}

Supplementary materials can be accessed at: http://www.mdpi.com/1424-8220/15/10/24791/s1.

\section{Acknowledgments}

The authors wish to thank the reviewers and editors for the careful review and the effort in processing this paper. This work was supported in part by the Program for Changjiang Scholars and Innovative Research Team in University (IRT1299), the National Natural Science Foundation of China (61301126 and 61471077), the Special Fund of Chongqing Key Laboratory (CSTC), the Fundamental and Frontier Research Project of Chongqing (cstc2013jcyjA40041 and cstc2013jcyjA40032), the Science Foundation for Young Scientists of CQUPT(A2012-77) and the Student Research Training Program of CQUPT (A2013-64). 


\section{Author Contributions}

The work presented in this paper corresponds to a collaborative development by all authors. Mu Zhou defined the research line and was involved in writing the manuscript. Qiao Zhang developed the proposed system, performed the data analysis and wrote the manuscript. Kunjie Xu and Zengshan Tian supervised the work. Yanmeng Wang and Wei He were involved in writing the manuscript. All authors have read, revised and approved the final manuscript.

\section{Conflicts of Interest}

The authors declare no conflict of interest.

\section{Appendix}

\section{Proof of the Scoring Function}

The flow chart of the proofs is shown in Figure A1.

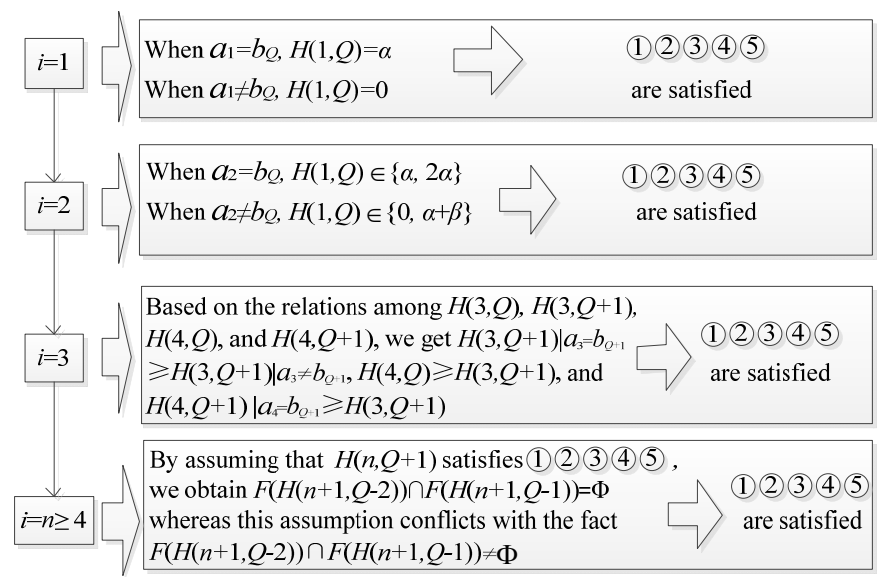

Figure A1. Flow chart of the proofs.

(1) Condition 1: $i=1$.

There are two cases to be considered as follows.

Case 1: $a_{1}=b_{1}$;

Case 2: $a_{1} \neq b_{1}$.

In Case 1 , we have $H(1,1)=\max \{0, \alpha\}=\alpha$. Then, if $j=2$ and $a_{1} \neq b_{2}$, we have $H(1,2)=$ $\max \left\{0, \beta, \max \left\{H(1-k, 2)+w_{k}\right\}, \max \left\{H(1,2-l)+w_{l}\right\}\right\}=0<\alpha$; ..; if $j=Q-1$ and $a_{1} \neq b_{Q-1}$, we have $H(1, Q-1)=\max \left\{0, \beta, \max \left\{H(1-k, Q-1)+w_{k}\right\}, \max \left\{H(1, Q-1-l)+w_{l}\right\}\right\}=0<\alpha$; and if $j=Q$ and $a_{1}=b_{Q}$; we have $H(1, Q)=\max \left\{0, \alpha, \max \left\{H(1-k, Q)+w_{k}\right\}, \max \left\{H(1, Q-l)+w_{l}\right\}\right\}=$ $\alpha>0$.

In Case 2, we have $H(1,1)=\max \{0, \beta\}=0$. Then, if $j=2$ and $a_{1} \neq b_{2}$, we have $H(1,2)=$ $\max \{0, \beta\}=0 ; \ldots$; if $j=Q-1$ and $a_{1} \neq b_{Q-1}, H(1, Q-1)=\max \{0, \beta\}=0$; and if $j=Q$ and $a_{1}=b_{Q}, H(1, Q)=\max \left\{0, \alpha, \max \left\{H(1-k, Q)+w_{k}\right\}, \max \left\{H(1, Q-l)+w_{l}\right\}\right\}=\alpha>0$. 
Therefore, we can easily obtain that $H(1, j)$ satisfies (1)(2)(3)(4)(5) under Condition 1.

(2) Condition 2: $i=2$.

There are two cases to be considered as follows.

Case 1: $a_{2}=b_{1}$;

Case 2: $a_{2} \neq b_{1}$.

In Case 1 , we have $H(2,1)=\max \left\{0, \alpha, \max \left\{H(2-k, 1)+w_{k}\right\}, \max \left\{H(2,1-l)+w_{l}\right\}\right\}=\alpha$. Then, if $j=2$ and $a_{2} \neq b_{2}$, we have $H(2,2)=\max \left\{0, H(1,1)+\beta, \max \left\{H(2-k, 2)+w_{k}\right\}, \max \{H(2,2-\right.$ $\left.\left.l)+w_{l}\right\}\right\} \in\{0, \alpha+\beta\}<\alpha$; ..; if $j=Q-1$ and $a_{2} \neq b_{Q-1}$, we have $H(2, Q-1)=\max \{0, H(1, Q-2)+$ $\left.\beta, \max \left\{H(2-k, Q-1)+w_{k}\right\}, \max \left\{H(2, Q-1-l)+w_{l}\right\}\right\} \in\{0, \alpha+\beta\}<\alpha$; and if $j=Q$ and $a_{2}=b_{Q}$, we have $H(2, Q)=\max \left\{0, H(1, Q-1)+\alpha, \max \left\{H(2-k, Q)+w_{k}\right\}, \max \left\{H(2, Q-l)+w_{l}\right\}\right\} \in$ $\{\alpha, 2 \alpha\} \geq \alpha$.

Then, we can easily obtain that $H(2, j)$ satisfies (1). Furthermore, the relationship between $H(2, j)$ and $H(3, j+1)$ is equivalent to the relationship between $H(1, j-1)$ and $H(2, j)$. Thus, based on the results in Condition 1, if $a_{1}=b_{j-1}$ and $a_{2}=b_{j}$, we have $H(1, j-1)=\alpha<2 \alpha=H(2, j)$; and if $a_{1} \neq b_{j-1}$ and $a_{2}=b_{j}$, we have $H(1, j-1)=0<\alpha=H(2, j)$. Hence, $H(2, j)$ satisfies (3) (5). On the other hand, if $a_{1}=b_{j-1}$ and $a_{2} \neq b_{j}$, we have $H(2, j) \in\{0, \alpha+\beta\}<\alpha=H(1, j-1)$; and if $a_{1} \neq b_{j-1}$ and $a_{2} \neq b_{j}$, we have $H(2, j)=0=H(1, j-1)$. Hence, $H(2, j)$ satisfies (2)(4).

In Case 2, we have $H(2,1)=\max \left\{0, \beta, \max \left\{H(2-k, 1)+w_{k}\right\}, \max \left\{H(2,1-l)+w_{l}\right\}\right\}=0$. Then, if $j=2$ and $a_{2} \neq b_{2}$, we have $H(2,2)=\max \left\{0, H(1,1)+\beta, \max \left\{H(2-k, 2)+w_{k}\right\}, \max \{H(2,2-\right.$ $\left.\left.l)+w_{l}\right\}\right\} \in\{0, \alpha+\beta\} ; \ldots$; if $j=Q-1$ and $a_{2} \neq b_{Q-1}$, we have $H(2, Q-1)=\max \{0, H(1, Q-2)+$ $\left.\beta, \max \left\{H(2-k, Q-1)+w_{k}\right\}, \max \left\{H(2, Q-1-l)+w_{l}\right\}\right\} \in\{0, \alpha+\beta\}$; and if $j=Q$ and $a_{2}=b_{Q}$, we have $H(i, j)=\max \left\{0, H(i-1, j-1)+\alpha, \max \left\{H(i-k, j)+w_{k}\right\}, \max \left\{H(i, j-l)+w_{l}\right\}\right\} \in$ $\{\alpha, 2 \alpha\}>\{0, \alpha+\beta\}$.

Then, we can easily obtain that $H(2, j)$ satisfies (1). Similarly, we equate the relationship between $H(2, j)$ and $H(3, j+1)$ to the relationship between $H(1, j-1)$ and $H(2, j)$. Based on the results in Condition 1, if $a_{1}=b_{j-1}$ and $a_{2}=b_{j}$, we have $H(1, j-1)=\alpha<2 \alpha=H(2, j)$; and if $a_{1} \neq b_{j-1}$ and $a_{2}=b_{j}$, we have $H(1, j-1)=0<\alpha=H(2, j)$. Hence, $H(2, j)$ satisfies (3)(5). On the other hand, if $a_{1}=b_{j-1}$ and $a_{2}=b_{j}$, we have $H(2, j) \in\{0, \alpha+\beta\}<\alpha=H(1, j-1)$; and if $a_{1} \neq b_{j-1}$ and $a_{2} \neq b_{j}$, we have $H(2, j) \geq 0=H(1, j-1)$. Hence, $H(2, j)$ satisfies (2) (4).

Therefore, we obtain that $H(2, j)$ satisfies (1)(2)(3)(4) under Condition 2.

In the results that follow, we only focus on the situation that $j \geq i$ in $H(i, j)$. Similar results can be easily obtained with respect to the $j<i$ in the $H(i, j)$ situation.

(3) Condition 3: $i=3$.

In this condition, if $j=3$ and $a_{3} \neq b_{3}$, we have $H(3,3)=\max \{0, H(2,2)+\beta, \max \{H(3-k, 3)+$ $\left.\left.w_{k}\right\}, \max \left\{H(3,3-l)+w_{l}\right\}\right\}=\max \{0, H(2,2)+\beta, H(2,3)+\beta-\alpha\} \in\{0, \alpha+\beta, 2 \alpha+\beta\} ;$ if $j=4$ and $a_{3} \neq b_{4}$, we have $H(3,4)=\max \left\{0, H(2,3)+\beta, \max \left\{H(3-k, 4)+w_{k}\right\}, \max \left\{H(3,4-l)+w_{l}\right\}\right\}=$ $\max \{0, H(2,3)+\beta, H(3,3)+\beta-\alpha\} \in\{0, \alpha+\beta, 2 \alpha+\beta, \alpha+2 \beta\} ; \ldots ;$ if $j=Q-1$ and $a_{3} \neq b_{Q-1}$, we have $H(3, Q-1)=\max \left\{0, H(2, Q-2)+\beta, \max \left\{H(3-k, Q-1)+w_{k}\right\}, \max \left\{H(3, Q-1-l)+w_{l}\right\}\right\} \in$ $\{0, \alpha+\beta, 2 \alpha+\beta, \alpha+2 \beta\}$; if $j=Q$ and $a_{3}=b_{Q}$, we have $H(3, Q)=\max \{0, H(2, Q-1)+$ $\left.\alpha, \max \left\{H(3-k, Q)+w_{k}\right\}, \max \left\{H(3, Q-l)+w_{l}\right\}\right\}=\max \{0, H(2, Q-1)+\alpha\} \geq H(3, Q-1)$; 
and if $j=Q+1$ and $a_{3} \neq b_{Q+1}$, we have $H(3, Q+1)=\max \{0, H(2, Q)+\beta, \max \{H(3-k, Q+1)+$ $\left.\left.w_{k}\right\}, \max \left\{H(3, Q+1-l)+w_{l}\right\}\right\}$. To obtain the relationship between $H(3, Q)$ and $H(3, Q+1)$, we need to examine the relations of four neighboring elements, $H(3, Q), H(3, Q+1), H(4, Q)$ and $H(4, Q+1)$. If $a_{4}=b_{Q}$, the calculation of $H(4, Q)$ is equivalent to the calculation of $H(3, Q+1)$ with $a_{3}=b_{Q+1}$. Since $\left.H(3, Q+1)\right|_{a_{3}=b_{Q+1}}>\left.H(3, Q+1)\right|_{a_{3} \neq b_{Q+1}}$, we have $H(4, Q) \geq H(3, Q+1)$; and if $a_{4}=b_{Q+1}$, we have $H(4, Q+1)=\max \left\{0, H(3, Q)+\alpha, \max \left\{H(4-k, Q+1)+w_{k}\right\}, \max \left\{H(4, Q+1-l)+w_{l}\right\}\right\}=$ $\max \left\{0,2 \alpha+H(2, Q-1), \max \left\{H(4-k, Q+1)+w_{k}\right\}, \max \left\{H(4, Q+1-l)+w_{l}\right\}\right\} \geq 2 \alpha+\beta \geq$ $H(3, Q+1)$.

Therefore, we can easily obtain that $H(3, j)$ satisfies (1)(2)(3)(4)(5) under Condition 3.

(4) Condition 4: $i=n \geq 4$.

In this condition, if $j=n$ and $a_{n} \neq b_{n}$, we have $H(n, n)=\max \{0, H(n-1, n-1)+\beta, \max \{H(n-$ $\left.\left.k, n)+w_{k}\right\}, \max \left\{H(n, n-l)+w_{l}\right\}\right\} \in\{0, \alpha+(n-1) \beta, \alpha+(n-2) \beta, \ldots, \alpha+\beta, 2 \alpha+(n-2) \beta, \ldots, 2 \alpha+$ $\beta, \ldots(n-1) \alpha+\beta\} ; \ldots$; if $j=Q-1$ and $a_{n} \neq b_{Q-1}$, we have $H(n, Q-1)=\max \{0, H(n-$ $\left.1, Q-2)+\beta, \max \left\{H(n-k, Q-1)+w_{k}\right\}, \max \left\{H(n, Q-1-l)+w_{l}\right\}\right\} \in\{0, \alpha+(n-1) \beta, \alpha+$ $(n-2) \beta, \ldots, \alpha+\beta, 2 \alpha+(n-2) \beta, \ldots, 2 \alpha+\beta, \ldots(n-1) \alpha+\beta\}$; if $j=Q$ and $a_{n}=b_{Q}$, we have $H(n, Q)=\max \left\{0, H(n-1, Q-1)+\alpha, \max \left\{H(n-k, Q)+w_{k}\right\}, \max \left\{H(n, Q-l)+w_{l}\right\}\right\}$; and if $j=Q+1$ and $a_{n} \neq b_{Q+1}$, we assume that $H(n, Q+1)$ satisfies (1)(2)(3)(4).

Then, under the $a_{n+1} \neq b_{Q-1}$ and $a_{n+1}=b_{Q}$ conditions, if $H(n+1, Q)<H(n+1, Q-1)$, we obtain:

$$
\max \left\{\begin{array}{c}
0 \\
\{H(n+1, Q-1)- \\
\alpha+\beta\} \\
H(n, Q-1)+\alpha \\
H(n, Q)-\alpha+\beta
\end{array}\right\}<\max \left\{\begin{array}{c}
0 \\
\{H(n+1, Q-2)- \\
\alpha+\beta\} \\
H(n, Q-1)+\beta \\
H(n, Q-1)-\alpha+\beta
\end{array}\right\}
$$

Based on Equation (11), since under the $a_{n} \neq b_{Q-2}, a_{n}=b_{Q-1}$ and $a_{n} \neq b_{Q}$ conditions, we have $H(n, Q-1) \geq H(n, Q-2)$ and $H(n, Q-1) \geq H(n, Q)$, Equation (11) can be simplified into $H(n+$ $1, Q-2)>H(n+1, Q-1)$. Thus, the range of the value $H(n+1, Q-2), F(H(n+1, Q-2))$, is not overlapped with the range of the value $H(n+1, Q-1), F(H(n+1, Q-1))$, notated as $F(H(n+$ $1, Q-2)) \cap F(H(n+1, Q-1))=\Phi$.

On the other hand, the relations of $H(n+1, Q-2) \in\{0, \alpha, \alpha+\beta, \ldots, \alpha+n \beta, 2 \alpha, 2 \alpha+\beta, \ldots, 2 \alpha+(n-$ 1) $\beta, n \alpha, n \alpha+\beta,(n+1) \alpha\}$ and $H(n+1, Q-1) \in\{0, \alpha+\beta, \ldots, \alpha+n \beta, 2 \alpha+\beta, \ldots, 2 \alpha+(n-1) \beta, n \alpha+\beta\}$ indicate that $F(H(n+1, Q-2)) \cap F(H(n+1, Q-1)) \neq \Phi$, which conflicts with the previous assumption in (Equation (11)). Therefore, we obtain that $H(n+1, Q) \geq H(n+1, Q-1)$, and thereby, $H(n+1, Q)$ satisfies (1)(2)(3)(4) under Condition 4.

In conclusion, it is proven that the definition of $H(i, j)$ satisfies (1)(2)(3)(4)(5).

\section{References}

1. Pei, L.; Chen, R.; Liu, J.; Kuusniemi, H. Using inquiry-based bluetooth rssi probability distributions for indoor positioning. J. Glob. Position. Syst. 2010, 9, 122-130.

2. Kim, S.J.; Kim, B.K. Dynamic ultrasonic hybrid localization system for indoor mobile robots. IEEE Trans. Ind. Electron. 2013, 60, 4562-4573. 
3. Chang, S.R.; Lin, W.; Chang, S.; Tu, C.; Wei, C.; Chien, C.; Tsai, C.; Chen, J.; Chen, A. A dual-band RF transceiver for multistandard WLAN applications. IEEE Trans. Microw. Theory Tech. 2005, 53, 1048-1055.

4. Lai, M.; Jeng, S. A microstrip three-port and four-channel multiplexer for WLAN and UWB coexistence. IEEE Trans. Microw. Theory Tech. 2005, 53, 3244-3250.

5. Yang, S.; Jung, E.; Han, S.K. Indoor location estimation based on LED visible light communication using multiple optical receivers. IEEE Commun. Lett. 2013, 17, 1834-1837.

6. Liu, M.; Qiu, K.; Che, S.; Li, S.; Hussain, B.; Wu, L.; Yue, C. Towards indoor localization using visible light Communication for consumer electronic devices. In Proceedings of the IEEE/RSJ International Conference in Intelligent Robots and Systems, Chicago, IL, USA, 14-18 September 2014; pp. 143-148.

7. Vegni, A.M.; Biagi, M. An indoor localization algorithm in a small-cell LED-based lighting system. In Proceedings of the International Conference on Indoor Positioning and Indoor Navigation, Sydney, NSW, Australia, 13-15 November 2012; Volume 147, pp. 1-7.

8. Ma, L.; Xu, Y. Received Signal Strength Recovery in Green WLAN Indoor Positioning System Using Singular Value Thresholding. Sensors 2015, 15, 1292-1311.

9. Du, Y.; Yang, D.; Xiu, C. Novel Method for Constructing a WIFI Positioning System with Efficient Manpower. Sensors 2015, 15, 8358-8381.

10. Sun, Y.; Xu, Y.; Li, C.; Ma, L. Kalman/Map filtering-aided fast normalized cross correlation-based Wi-Fi fingerprinting location sensing. Sensors 2013, 13, 15513-15531.

11. Zhou, M.; Wang, A.K.; Tian, Z.; Luo, X.; Xu, K.; Shi, R. Personal Mobility Map Construction for Crowd-Sourced Wi-Fi Based Indoor Mapping. IEEE Commun. Lett. 2014, 18, 1427-1430.

12. Chen, Y.; Fracisco, J.A.; Trappe, W.; Martin, R.P. A parctical approach to landmark deployment for indoor localization. In Proceedings of the 3rd Annual IEEE Communications Society on Sensor and Ad Hoc Communications and Networks, Reston, VA, USA, 28 September 2006; pp. 3429-3443.

13. Zhou, M.; Wang, A.K.; Tian, Z.; Zhang, V.Y.; Yu, X.; Luo, X. Adaptive Mobility Mapping for People Tracking Using Unlabelled Wi-Fi Shotgun Reads. IEEE Commun. Lett. 2013, 17, 87-90.

14. Zhou, M.; Xu, Y.; Ma, L.; Tian, S. On the statistical errors of RADAR location sensor networks with built-in Wi-Fi Gaussian linear fingerprints. Sensors 2012, 12, 3605-3626.

15. Ma, L.; Xu, Y.; Wu, D. A novel two-step WLAN indoor positioning method. J. Comput. Inf. Syst. 2010, 6, 4627-4636.

16. Wang, B.; Zhou, S.; Liu, W.; Mo, Y. Indoor localization based on curve fitting and location search using received signal strength. IEEE Trans. Ind. Electron. 2015, 62, 572-582.

17. Zhou, M.; Tian, Z.; Xu, K.; Yu, X.; Hong, X.; Wu, H. SCaNME: Location Tracking System in Large-Scale Campus Wi-Fi Environment Using Unlabeled Mobility Map. Expert Syst. Appl. 2014, 41, 3429-3443.

18. Bahl, P.; Padmanabhan, V.N. RADAR: An in-building RF-based user location and tracking system. In Proceedings of the IEEE INFOCOM, Tel Aviv, Israel, 26-30 March 2000; Volume 2, pp. 775-784. 
19. Youssef, M.; Agrawala, A. The Horus WLAN location determination system. In Proceedings of the 3rd International Conference on Mobile Systems, Applications, and Services, Seattle, WA, USA, 6-8 June 2005; pp. 205-218.

20. Kaemarungsi, K.; Krishnamurthy, P. Analysis of WLAN's received signal strength indication for indoor location fingerprinting. Pervasive Mob. Comput. 2012, 8, 292-316.

21. Zhou, M.; Tian, Z.; Xu, K.; Yu, X.; Wu, H. Theoretical Entropy Assessment of Fingerprint-based Wi-Fi Localization Accuracy. Expert Syst. Appl. 2013, 40, 6136-6149.

22. Wu, C.; Yang, Z.; Liu, Y.; Xi, W. WILL: Wireless indoor localization without site survey. IEEE Trans. Parallel Distrib. Syst. 2013, 24, 839-848.

23. Shin, H.; Chon, Y.; Cha, H. Unsupervised construction of an indoor floor plane using a smartphone. IEEE Trans. Syst. Man Cybern. Part C Appl. Rev. 2012, 42, 889-898.

24. Shin, H.; Cha, H. Wi-Fi fingerprint-based topological map building for indoor user tracking. In Proceedings of the 16th IEEE International Conference on Embedded and Real-Time Computer Systems and Applications, Macau, China, 23-25 August 2010; pp. 105-113.

25. Hardegger, M.; Roggen, D.; Mazilu, S.; Troster, G. ActionSLAM: Using location-related actions as landmarks in pedestrian SLAM. In Proceedings of the International Conference on Indoor Positioning and Indoor Navigation, Sydney, Australia, 13-15 November 2012; pp. 1-10.

26. Bruno, L.; Robertson, P. WiSLAM: Improving footSLAM with WiFi. In Proceedings of the International Conference on Indoor Positioning and Indoor Navigation, Guimaraes, Portugal, 21-23 September 2011; pp. 1-10.

27. Kao, W.; Huy, B. Indoor navigation with smartphone-based visual SLAM and bluetooth-connected wheel-robot. In Proceedings of the International Automatic Control Conference, Nantou, Taiwan, 2-4 December 2013; pp. 395-400.

28. Huang, J.; Millman, D.; Quigley, M.; Stavens, D.; Thrun, S.; Aggarwal, A. Efficient, generalized indoor WiFi graphSLAM. In Proceedings of the IEEE International Conference on Robotics and Automation, Beijing, China, 7-10 August 2011; pp. 1038-1043.

29. Mirowski, P.; Tin, K.H.; Saehoon, Y.; Macdonald, M. SignalSLAM: Simutanous localization and mapping with mixed WiFi, bluetooth, LTE and magnetic signals. In Proceedings of the International Conference on Indoor Positioning and Indoor Navigation, Montbéliard, France, 28-31 October 2013; pp. 1-10.

30. Hussin, Z. Fast-converging indoor mapping for wireless indoor localization. In Proceedings of the 7th Annual PhD Forum on Pervasive Computing and Communications, Budapest, Hungary, 24-28 March 2014; pp. 171-173.

31. Bisio, I.; Lavagetto, F.; Marchese, M.; Sciarrone, A. GPS/HPS-and Wi-Fi Fingerprint-Based Location Recognition for Check-in Applications over Smartphones in Cloud-Based LBSs. IEEE Trans. Multimed. 2013, 15, 858-869.

32. Bisio, I.; Cerruti, M.; Lavagetto, F.; Marchese, M.; Pastorino, M.; Randazzo, A. A Trainingless Wi-Fi Fingerprint Positioning Approach Over Mobile Devices. IEEE Antennas Wirel. Propag. Lett. 2014, 13, 832-835.

33. Sanchez, R.D.; Hernandez, M.P.; Quinteiro, J.M.; Alonso, G.I. A Low Complexity System Based on Multiple Weighted Decision Trees for Indoor Localization. Sensors 2015, 15, 14809-14829. 
34. Youssef, M.A.; Agrawala, A.; Shankar, A.U. WLAN Location Determination via Clustering and Probability Distributions. In Proceedings of the IEEE PerCom, Fort Worth, TX, USA, 26 March 2003; pp. 143-152.

35. Severi, S.; Liva, G.; Chiani, M.; Dardari, D. A New Low-Complexity User Tracking Algorithm for WLAN-Based Positioning System. In Proceedings of the 16th IST Mobile and Wireless Communications Summit, Budapest, Hungary, 15-17 December 2007; pp. 1-5.

36. Pietro, R.; Rocco, R.; Paolo, A. A Computationally Approach to WLAN localization based on multiple filters. In Proceedings of the International Conference on Localization and GNSS, Gothenburg, Sweden, 22-24 June 2015; pp. 1-6.

37. Bisio, I.; Lavagetto, F.; Marchese, M.; Sciarrone, A. Performance comparison of a probabilistic fingerprint-based indoor positioning system over different smartphone. In Proceedings of the International Symposium on Performance Evaluation of Computer and Telecommunication Systems, Toronto, Canada, 7-10 July 2013; pp. 161-166.

38. Bisio, I.; Lavagetto, F.; Marchese, M.; Sciarrone, A. Energy efficient WiFi-based fingerprinting for indoor positioning with smartphones. In Proceedings of the IEEE GLOBECOM, Atlanta, GA, USA, 9-13 December 2013; pp. 4639-4643.

39. Smith, T.F.; Waterman, M.S. Identification of Common Molecular Subsequences. J. Mol. Biol. 1981, 147, 195-197.

(c) 2015 by the authors; licensee MDPI, Basel, Switzerland. This article is an open access article distributed under the terms and conditions of the Creative Commons Attribution license (http://creativecommons.org/licenses/by/4.0/). 